\title{
An Alternative Variational Framework for Image Denoising
}

\author{
Elisha Achieng Ogada, ${ }^{1,2}$ Zhichang Guo, ${ }^{1}$ and Boying Wu ${ }^{1}$ \\ ${ }^{1}$ Department of Mathematics, Harbin Institute of Technology, Harbin, 150001, China \\ ${ }^{2}$ Department of Mathematics, Egerton University, Egerton, Kenya
}

Correspondence should be addressed to Zhichang Guo; mathgzc@gmail.com

Received 13 March 2014; Accepted 6 April 2014; Published 5 May 2014

Academic Editor: Carlos Lizama

Copyright (c) 2014 Elisha Achieng Ogada et al. This is an open access article distributed under the Creative Commons Attribution License, which permits unrestricted use, distribution, and reproduction in any medium, provided the original work is properly cited.

\begin{abstract}
We propose an alternative framework for total variation based image denoising models. The model is based on the minimization of the total variation with a functional coefficient, where, in this case, the functional coefficient is a function of the magnitude of image gradient. We determine the considerations to bear on the choice of the functional coefficient. With the use of an example functional, we demonstrate the effectiveness of a model chosen based on the proposed consideration. In addition, for the illustrative model, we prove the existence and uniqueness of the minimizer of the variational problem. The existence and uniqueness of the solution associated evolution equation are also established. Experimental results are included to demonstrate the effectiveness of the selected model in image restoration over the traditional methods of Perona-Malik (PM), total variation (TV), and the D- $\alpha$-PM method.
\end{abstract}

\section{Introduction}

The objective of any image restoration process should not focus only on the removal of noise, but it should also observe, as Perona and Malik [1], Koenderink [2], and Witkin [3] determined, that no new spurious details are created in the restored image; at each scale-space representation, the boundaries/edges are sharp or preserved, and, at all scales, intraregion smoothing is preferred to interregion smoothing.

In the light of the above considerations, researchers have observed that it is then logical to obtain or develop edge indicators that would be adapted to the local image structure [4]. Consequently, a number of edge indicators have been proposed and logically grafted into the partial differential equation (PDE) based evolution equations $[1,5]$. In addition, energy minimization problems are continually being formulated which focus on producing adaptive partial differential equations $[6,7]$.

Total variation (TV) method, widely considered a powerful technique for smoothing, edge preservation, and general image restoration, was first proposed by Rudin et al. [8]. The method is based on the strength of the argument that TV norms principally are $L^{1}$-norms of derivatives and that $L^{1}$ norms provide the proper basis for image restoration $[8,9]$.
TV functionals are defined in the space of functions of bounded variation (BV) and therefore do not necessarily require image functions to be continuous and smooth. This fact makes them allow for "jumps" or discontinuities and thus be able to protect edges.

The $L^{2}$-norm of the gradient of image allows removing noise; however, it has the adverse effect of penalizing too much the gradients corresponding to edges [10]. A functional based on the $L^{2}$-norm does not permit discontinuities in the solution, and thus edges cannot be recovered properly [11].

The total variation (TV) norm in [8] is a regularization functional of the form

$$
\operatorname{TV}(u)=\int_{\Omega}|\nabla u| d x=\int_{\Omega} \sqrt{u_{x}^{2}+u_{y}^{2}} d x .
$$

Although TV regularization above allows for edge recovery, it has some demerits. Firstly, the formulation favours solutions which are piecewise constant. This has the effect of causing staircase effects and may even generate false edges on the image [12]. Secondly, the method has the effect of reducing contrast even in regions of the same pixel intensity or in noise-free observed images $[13,14]$.

Various modifications have, therefore, been proposed, in an attempt to address the drawbacks of the TV model, to 
make it as adaptive as possible to the local image structure. For instance, Strong and Chan in [15] proposed the spatially adaptive regularization functional of the form

$$
J(u)=\int_{\Omega} \alpha(x)|\nabla u| d x
$$

where the control factor $\alpha(x)$ is designed to slow diffusion in the neighbourhood of edges. Then, we have the efforts, according to Blomgren et al. in [13], which produced a denoising functional of the form

$$
J(u)=\int_{\Omega}|\nabla u|^{p(|\nabla u|)},
$$

where $p$ is a nondecreasing function of the magnitude of gradient, $p(s) \rightarrow 2$ as $s \rightarrow 0$ and $p(s) \rightarrow 1$ as $s \rightarrow \infty$. This model is designed to automatically tap into the benefits of both isotropic diffusion and TV regularization. For other modifications, we refer the reader to, among others, the works in $[12,16,17]$.

In this paper, however, we propose an alternative framework of variational model for image denoising, where the regularization potential is a product of a gradient based functional coefficient and the norm of gradient of image (potential function for TV); namely, $F(u)=\int_{\Omega} \Psi(|\nabla u|) \cdot|\nabla u| d x$. That is, the coefficient function is a function of the magnitude of the gradient of the image. We propose the criteria for the choice of the coefficient. Finally, we have selected a model based on the proposed criteria, as an example for further analysis and demonstration of experimental results.

The structure of this paper is as follows. In Section 2, we present the proposed model (4) and discuss the general criteria for choosing the functional coefficient to the traditional TV potential. Section 2 is concluded by considering, based on the proposed criteria, an example functional for further analysis. In Section 3, we give certain preliminary definitions and lemmas we rely on, variously, in this paper. In addition, we prove the existence and uniqueness of the solution to the minimization problem (11) and the associated evolution equation (27)-(29). In Section 4, we define the weak solution to the evolution problem (27)-(11). Furthermore, we present the formulation of the approximate evolution equation (32)(36), prove the existence of solutions to the approximate evolution problem, and conclude Section 4 with the existence and uniqueness of the solution to the evolution problem (27)-(29). In Section 5, we give the numerical schemes and experimental results to demonstrate the strength and effectiveness of our method. Additionally, we have presented a brief discussional comparison of our results with those of other methods like PM, original TV, and D- $\alpha$-PM method by Guo et al. [5]. A brief summary concludes the paper in Section 6 .

\section{Proposed Model}

In this section, inspired by the works of Chan and Shen [18], Vese [19], and Chen et al. [12], among others, we propose an alternative framework for total variation based denoising model. The model is based on minimization of a functional, where the regularization potential is a product of the total variation potential and a gradient based functional coefficient. The coefficient, which acts to penalize the norm of gradient and detect any edges, is also taken as function of the norm of the gradient of image. So, we present the general form of the model and determine certain properties of the functional coefficient, especially in terms of linearity, sublinearity, and superlinearity growth at infinity. Ultimately, we have selected a specific example of model, based on the proposed guidelines, for further analysis.

2.1. The New Framework for Energy Functional. The proposed energy functional is given in the following general form:

$$
\min _{u \in \mathrm{BV}^{2}(\Omega)}\left\{F(u)=\int_{\Omega} \Psi(|\nabla u|)|\nabla u| d x+\frac{\lambda}{2} \int_{\Omega}(u-f)^{2} d x\right\},
$$

where $\Psi(s)$ is a function that detects edges and penalizes the norm of gradient and $f$ is the noise image. Observe that if we set $\Psi(s)=s$, then the kernel of $F(u)$ becomes a variational problem of the $L^{2}$-norm that is known not to allow for discontinuities, since it leads to the traditional edge obliterating isotropic diffusion [20], while if $\Psi(s)=1$ the problem becomes the usual TV functional, which allows a diffusion mechanism that is strictly normal to the image gradient [12].

The functional $F(u)$ in (4) should verify the EulerLagrange equation

$$
0=-\operatorname{div}\left\{\left(\Psi^{\prime}(|\nabla u|)+\frac{\Psi(|\nabla u|)}{|\nabla u|}\right) \nabla u\right\}+\lambda(u-f) .
$$

Next, we establish certain properties of $\Psi(s)$ from (5) that should influence its choice. For the purposes of denoising, to be able to smooth the image in homogeneous regions, that is, regions where $s=|\nabla u| \rightarrow 0, \Psi(s)$ is expected such that $\lim _{s \rightarrow 0}\left(\Psi^{\prime}(|\nabla u|)+(\Psi(|\nabla u|) /|\nabla u|)\right)=C$ (constant) $>0$. On the other hand, to preserve discontinuities (edges) in the image, that is, in areas where $s=|\nabla u| \rightarrow+\infty, \Psi(s)$ takes a form such that $\lim _{s \rightarrow+\infty}\left(\Psi^{\prime}(|\nabla u|)+(\Psi(|\nabla u|) /|\nabla u|)\right)=0$.

Now, for a little more precision on the expected behaviour of $\Psi(s)$, we decompose divergence part in terms of the tangent and normal directions to the isophote lines. Equation (5) then becomes

$$
\begin{aligned}
0= & {\left[\Psi^{\prime}(|\nabla u|)+\frac{\Psi(|\nabla u|)}{|\nabla u|}\right] u_{T T} } \\
& +\left[2 \Psi^{\prime}(|\nabla u|)+\Psi^{\prime \prime}(|\nabla u|)|\nabla u|\right] u_{N N}-\lambda(u-f) .
\end{aligned}
$$

Then, to be able to achieve isotropic diffusion within homogeneous regions, from (6), we may impose the condition that

$$
\lim _{s \rightarrow 0}\left[\Psi^{\prime}(s)+\frac{\Psi(s)}{s}\right]=\lim _{s \rightarrow 0}\left[2 \Psi^{\prime}(s)+\Psi^{\prime \prime}(s) \cdot s\right]=C>0,
$$

where $C$ is a constant. The above condition yields from (6) a diffusion model that diffuses isotropically. 
In the regions neighboring the edges, the model should dissipate diffusion effects across the edges and favor diffusion along the edges. This implies that as $s \rightarrow \infty$, $\left[\Psi^{\prime}(s)+\right.$ $(\Psi(s) / s)] \rightarrow C_{1}$, where $C_{1}$ is an arbitrary positive constant, while $\left[2 \Psi^{\prime}(s)+\Psi^{\prime \prime}(s) \cdot s\right] \rightarrow 0$. These conditions might be difficult to meet simultaneously for $\Psi(s)$. Therefore, motivated by the works in $[10,19]$, a weaker compromise is imposed by demanding that both terms approach zero, but at different rates, with diffusion along the direction normal to the isophote lines approaching zero faster than the diffusion along the tangent to isophote lines. This leads to the condition that

$$
\lim _{s \rightarrow+\infty} \frac{\left[2 \Psi^{\prime}(s)+\Psi^{\prime \prime}(s) \cdot s\right]}{\left[\Psi^{\prime}(s)+(\Psi(s) / s)\right]}=0
$$

Hence, we observe that, for $\Psi(s)$ to become an effective coefficient in the functional in (4), it should be such that conditions (7) and (8) are satisfied.

For simplicity of notation in subsequent stages, we will denote, from (5),

$$
\begin{aligned}
& \varphi^{\prime}(\nabla u):=\Psi^{\prime}(|\nabla u|)|\nabla u|+\Psi(|\nabla u|), \\
& \text { so that } \varphi(\nabla u):=\Psi(|\nabla u|)|\nabla u| .
\end{aligned}
$$

Furthermore, we are interested in the properties of $\Psi(s)$ with regard to its growth at infinity and its overall impact on the functional $F(u)$ :

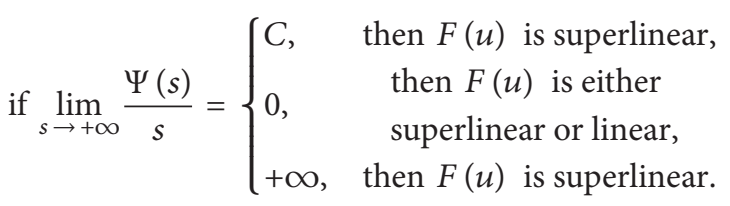

Note that $C>0$ is a constant and growth.

2.1.1. Summary on the Characterization of Growth of $\Psi(\cdot)$. The aim of this section is to discuss the property of the function coefficient $\Psi(s)$ that will lead to a functional $F(u)$ of linear growth at infinity, but, of course, subject to conditions (7)-(8). The characterization is based upon the results of process (10).

Observe that setting the coefficient $\Psi(s)$ in the regularization kernel, as a function of the norm of gradient of the image to be linear growth, leads to a functional $F(u)$ in (4), which is of superlinear growth. Such a functional yields associated diffusion equation which either diffuses images uniformly, without being sensitive to discontinuities or edges, or generally does not yield good denoising results [10, 21].

Choosing $\Psi(s)$ that is of superlinear growth leads to $F(u)$ which is invariably of superlinear growth. For instance, if we set $\Psi(s)=s^{p},(p>1)$ which is of superlinear growth at infinity, then we obtain the function $\Psi(s) \cdot s=s^{p+1}$. This functional is of superlinear growth and does not give good results in image denoising. This is because, with such a choice of $\Psi(s)$, the derivative of $\Psi(s) \cdot s$ would yield a nondegenerate elliptic differential operator of the second order, which would have an oversmoothing effect under the optimality condition [22].
Bildhauer and Fuchs [21] reckon that a regularization function need not necessarily be of power growth. Therefore, another example for $\Psi(s)$ of superlinear growth is $\Psi(s) \cdot s=$ $s^{2} \log (1+s)$, where $\Psi(s)=s \log (s+1)$ is viewed as some kind of compromise between the cases $p>1$ and $p=1$ of coefficient of power growth [21]. This formulation too does not promise any better results.

Consequently, we observe that to achieve maximal results, which entails reconstructing a noise image in such a way that the edges are protected while diffusion within homogeneous regions proceeds rather uniformly, it is required that $\Psi(s)$ be of sublinear growth at infinity. Also, the chosen $\Psi(s)$ should be such that the functional $F(u)$ generated is of linear growth. Moreover, $\Psi(s)$ should be such that the conditions in (7)-(8) are satisfied. Functionals which arise from such potential functions tend to give better image denoising results. Some examples of the functions $\Psi(s)$ of sublinear growth are $\Psi(s):=s /(1+s), \Psi(s):=\log (1+s)$, and $\Psi(s):=1 /(1+\alpha s)$. The function $\Psi(s):=s /(1+s)$ is a good candidate for further consideration.

In this paper, therefore, we choose the control function $\Psi(s):=s /(1+s)$, which is function of sublinear growth at infinity. This leads to a nondecreasing potential function for $s \in \mathbb{R}^{+}$, which is strictly convex and of linear growth at infinity given in the form $\Psi(s) \cdot s:=s^{2} /(1+s)$.

With this choice, we observe the following further properties of $\Psi(s)$;

(i) $\lim _{s \rightarrow 0} \Psi(s)=0$;

(ii) $\lim _{s \rightarrow \infty} \Psi(s)=1$;

(iii) $\Psi(s)$ is nondecreasing for $s \geq 0$. This, as observed from (6), ensures only forward diffusion along the tangent to the isophote lines.

\section{The Minimization Problem}

Our model based on the presentation above is then given by

$$
\min _{u \in \operatorname{BV} \cap L^{2}(\Omega)}\left\{F(u)=\int_{\Omega} \varphi(\nabla u) d x+\frac{\lambda}{2} \int_{\Omega}(u-f)^{2} d x\right\},
$$

where

$$
\varphi(\nabla u):=\frac{|\nabla u|^{2}}{1+|\nabla u|} .
$$

Hence, in this section, we study the existence and uniqueness of the minimization problem (11). The following preliminary information prefaces our reasoning here and in the subsequent sections of this paper.

\subsection{Preliminaries}

Definition 1. Let $\Omega$ be an open subset of $\mathbb{R}^{n}$. A function $u \in$ $L^{1}(\Omega)$ has a bounded variation in $\Omega$, denoted by $\operatorname{BV}(\Omega)$, if

$$
\int_{\Omega}|D u|=\sup \left\{\int_{\Omega} u \operatorname{div} \varphi d x: \varphi \in C_{0}^{1}\left(\Omega ; \mathbb{R}^{n}\right),|\varphi| \leq 1\right\}<\infty \text {, }
$$


where $\mathrm{BV}(\Omega)$ denotes the space of such functions. Then, BVnorm is given by

$$
\|u\|_{\mathrm{BV}}=\int_{\Omega}|\nabla u|+\|u\|_{L^{1}(\Omega)} .
$$

Definition 2 (see [23]). If $u \in \mathrm{BV}(\Omega)$, then

$$
D u=\nabla u d x+D_{s} u .
$$

And writing $D u$ and its total variation on $\Omega,|D u|(\Omega)$, one has (see [7])

$$
|D u|(\Omega)=\int_{\Omega}|\nabla u| d x+\int_{\Omega} D_{s} u .
$$

$D u$ is a Radon measure, where $\nabla u$ is the density of the absolutely continuous part of $D u$ with respect to the Lebesgue measure, $\mathscr{L}^{n}$, and $D_{s} u$ is the singular part.

Lemma 3 (see [24]). Let $\varphi: \mathbb{R}^{n} \rightarrow \mathbb{R}$. The function $\varphi$ is convex if and only if

$$
\varphi(x) \geq \varphi^{\prime}(y)(x-y)+\varphi(y), \quad \forall x, y \in \mathbb{R}^{n},
$$

and if $\varphi \in C^{2}\left(\mathbb{R}^{2}\right)$, then $\varphi$ is convex if and only if $\nabla^{2} \varphi \geq 0$.

Lemma 4 (see [10]). Let $\Phi: \mathbb{R} \rightarrow \mathbb{R}^{+}$be convex, even, and nondecreasing on $\mathbb{R}^{+}$with linear growth at infinity. Also, let $\Phi^{\infty}$ be the recession function of $\Phi$ defined by

$$
\Phi^{\infty}(\omega)=\lim _{s \rightarrow \infty} \frac{\Phi(s \omega)}{s} .
$$

Then, for $u \in B V(\Omega)$ and setting $\Phi(\theta)=\Phi(|\theta|)$, one has

$$
\int_{\Omega} \Phi(D u)=\int_{\Omega} \Phi(|\nabla u|) d x+\Phi^{\infty}(1) \int_{\Omega} D_{s} u .
$$

This implies that $u \rightarrow \int_{\Omega} \Phi(D u)$ is lower semicontinuous for the $B V(\Omega)$ topology.

3.2. Existence and Uniqueness of Solution to the Minimization Problem. Since the objective here is to preserve edges, which are viewed as discontinuities, the natural function space to seek the solution is the $\mathrm{BV}(\Omega)$ space. In image analysis, the $\mathrm{BV}(\Omega)$ space allows for discontinuities across the edges of images. Furthermore, due to nonreflexivity of the $L^{1}(\Omega)$ space, which would have been a more natural space within which to seek the solution, it is noted that the solution to the minimization problem might not exist there. Therefore, the BV- $w^{*}(\Omega)$, which denotes BV-weak ${ }^{*}$ topology, provides the most reasonable alternative space for the existence of the solution. In what follows, we will, for convenience of notation, refer to $\mathrm{BV}-$ weak $^{*}(\Omega)$ simply as $\mathrm{BV}(\Omega)$. This space allows obtaining compactness because of the separability of $L^{1}(\Omega)$ space even though it is not reflexive [10]. Hence, we state and prove the following existence and uniqueness theorem for our problem.

Theorem 5. Given $\varphi(\nabla u)$ as assigned above, there exists $u \in$ $B V(\Omega) \cap L^{2}(\Omega)$ which is a unique solution to the minimization problem (11).
Proof. Since $\varphi(\nabla u)$ as specifically defined above for (11) is of linear growth and given the fact that $\lim _{s \rightarrow \infty} \varphi(s)=+\infty$, it implies that it is also coercive. Consequently, there exists a sequence $\left\{u_{n}\right\} \in \operatorname{BV}(\Omega) \cap L^{2}(\Omega)$ such that $F\left(u_{n}\right) \leq C$. Hence, we have $\int_{\Omega} \varphi\left(\nabla u_{n}\right)<C$, and, from the inequality of the second component of $F(u)$, namely, $\int_{\Omega}\left(u_{n}-f\right)^{2} d x \leq C$, if we define $w_{n}=(1 /|\Omega|) \int_{\Omega} u_{n} d x, v_{n}=u_{n}-w_{n}$, we observe that $\int_{\Omega} v_{n} d x=0$. We then deduce from [10] that $\left|\int_{\Omega} u_{n} d x\right| \leq C$ and $\left\|u_{n}\right\|_{L^{2}(\Omega)} \leq C$.

This indicates that $\left\{u_{n}\right\}$ is bounded in $L^{2}(\Omega)$ and $L^{1}(\Omega)$. And from $F\left(u_{n}\right) \leq C$ it is also clear that $u_{n}$ is abounded in $\operatorname{BV}(\Omega)$. Thus, there exists a subsequence $\left\{u_{n j}\right\} \in \operatorname{BV}(\Omega)$ of $\left\{u_{n}\right\}$ such that

$$
\begin{aligned}
& u_{n_{j}} \longrightarrow u, \quad \text { weakly in } L^{2}(\Omega), \\
& u_{n_{j}} \longrightarrow u, \quad \text { strongly in } L^{1}(\Omega) .
\end{aligned}
$$

And on the strength of the lemma on convex functions of measures (see Lemma 4) and the weak lower semicontinuity of the $L^{2}$-norm for the second component of $F(u)$, we deduce that $F(u)$ is lower semicontinuous. In fact, the recession function $\Phi^{\infty}(\omega)$ in the definition of convex function of measures is finite for our functional. That is, $\Phi^{\infty}(\omega)=1$. Hence, we obtain that

$$
\begin{aligned}
& \int_{\Omega} \varphi(\nabla u) d x+\int_{\Omega}(u-f)^{2} d x \\
& \quad \leq \liminf _{j \rightarrow \infty} \int_{\Omega} \varphi\left(\nabla u_{n_{j}}\right) d x+\liminf _{j \rightarrow \infty} \int_{\Omega}\left(u_{n_{j}}-f\right)^{2} d x
\end{aligned}
$$

or

$$
\begin{aligned}
\int_{\Omega} \varphi & (\nabla u) d x+\int_{\Omega}(u-f)^{2} d x \\
& \leq \liminf _{j \rightarrow \infty}\left\{\int_{\Omega} \varphi\left(\nabla u_{n_{j}}\right) d x+\int_{\Omega}\left(u_{n_{j}}-f\right)^{2} d x\right\} .
\end{aligned}
$$

We, thus, have

$$
F(u) \leq \liminf _{j \rightarrow \infty} F\left(u_{n_{j}}\right)=\min _{u \in \operatorname{BV}(\Omega) \cap L^{2}(\Omega)} F(u) .
$$

Thus, $u \in \operatorname{BV}(\Omega) \cap L^{2}(\Omega)$ is a minimizer of the problem (11). Uniqueness of $u$ is drawn from the strict convexity of $\varphi(\nabla u)$ and convexity of the second component of $F(u)$, which implies the overall strict convexity of the functional $F(u)$. Additionally, a strictly convex functional admits at most one minimum. This, then, implies that $u$ is a unique minimizer of (11).

3.3. The Associated Evolution Equation. From the energy minimization problem as assigned above, namely,

$$
\min _{u \in \mathrm{B} \vee \cap L^{2}(\Omega)}\left\{F(u)=\int_{\Omega} \frac{|\nabla u|^{2}}{1+|\nabla u|} d x+\frac{\lambda}{2} \int_{\Omega}(u-f)^{2} d x\right\},
$$


we have the associated Euler-Lagrange equation given by

$$
0=-\operatorname{div}\left(\frac{2+|\nabla u|}{(1+|\nabla u|)^{2}} \nabla u\right)+\lambda(u-f), \quad x \in \Omega,
$$

with the Neumann boundary condition

$$
\frac{\partial u}{\partial \vec{n}}=0, \quad x \in \partial \Omega .
$$

We compute for $u$ in the Euler-Lagrange equation (25) by putting it into a dynamical system, where the time $t$ is used as an evolution parameter. Hence, the evolution equation associated with the minimization problem (4) is given by

$$
\begin{gathered}
\frac{\partial u}{\partial t}=\operatorname{div}\left(\frac{2+|\nabla u|}{(1+|\nabla u|)^{2}} \nabla u\right)-\lambda(u-f), \quad(x, t) \in Q_{T} \\
u(x, 0)=f(x), \quad x \in \Omega \\
\frac{\partial u}{\partial \vec{n}}=0, \quad(x, t) \in \partial Q_{T}
\end{gathered}
$$

where $f(x)$ is the noise image, $Q_{T}:=[0, T] \times \Omega$, and $\partial Q_{T}:=$ $\partial \Omega \times[0, T]$.

In order to see whether the potential $\varphi(s):=\Psi(s) \cdot s$, as defined above, respects the general principle of image reconstruction, where it is required that reconstructed image be formed by homogeneous regions separated by sharp edges, we decompose the divergence term of (25) using the local image structures like tangent and normal directions to the isophote lines. Writing it in its nonconservative form, analogous to (6), yields

$$
0=-\left(\frac{2+|\nabla u|}{(1+|\nabla u|)^{2}}\right) u_{T T}-\left(\frac{2}{(1+|\nabla u|)^{3}}\right) u_{N N}+\lambda(u-f) \text {. }
$$

Notice from (30) that as $|\nabla u| \rightarrow 0$, signaling homogeneous regions, the potential $\varphi(s)$ behaves like a linear isotropic diffusion encouraging uniform smoothing in both the $u_{N N}$ and $u_{T T}$ directions. However, as $|\nabla u| \rightarrow \infty$, corresponding to the neighbourhood of the edges, diffusion rate along the normal (or $u_{N N}$ ) direction is diminished, while the diffusion along the tangent (or $u_{T T}$ ) direction is preferred, thereby preserving the edges. The model therefore is well-behaved, since it reasonably satisfies the principle cited above.

\section{Weak Solution to the Flow Associated with the Minimization Problem}

In this section, we present the definition of weak solution to the evolution problem (27)-(29), propose an approximating evolution equation, establish existence result for the solution of the approximate evolution equation, and, then, by logical mathematical manipulation and passing to the limits, present a proof of the existence and uniqueness of the solution of the evolution problem (27)-(29).

We refer to the works in [25-28] as the motivation for our definition of the weak solution to the evolution problem (27)(29).
Definition 6. A measurable function $u: Q_{T} \rightarrow \mathbb{R}$, is called an entropy solution of (27)-(29) if $u \in L^{\infty}([0, T] ; B V(\Omega) \cap$ $\left.L^{2}(\Omega)\right), \partial u / \partial t \in L^{2}\left(Q_{T}\right), u(x, 0)=f$ in $\Omega$ in the trace sense, and if there exists $z \in L^{1}\left(Q_{T} ; \mathbb{R}^{N}\right),\|z\|_{L^{\infty}\left(Q_{T} ; \mathbb{R}^{N}\right)} \leq 1, \partial u / \partial t=$ $\operatorname{div}(z)-\lambda(u-f)$ in $\mathscr{D}^{\prime}\left(Q_{T}\right)$ such that

$$
\begin{aligned}
\int_{\Omega}(u(t)-v(t)) \frac{\partial u}{\partial t} d x \\
\leq \int_{\Omega} z(t) \cdot \nabla v d x-\|D u(t)\| \\
\quad-\lambda \int_{\Omega}(u-f)(u(t)-v) d x
\end{aligned}
$$

for every $v \in L^{\infty}\left(0, T ; W^{1,1}(\Omega)\right)$, a.e. for $t \in[0, T]$.

4.1. Existence of the Solution of the Approximate Evolution Problem (32)-(34). Before we study the existence and uniqueness of the evolution problem (27)-(29) above, let us consider the following approximate evolution problem: for $1<p \leq 2$ and $f_{p} \in W^{1, p}(\Omega)$, we construct the approximation

$$
\begin{gathered}
\frac{\partial u_{p}}{\partial t}=\operatorname{div}\left(\varphi_{p}^{\prime}\left(\nabla u_{p}\right)\right)-\lambda\left(u_{p}-f_{p}\right), \quad(x, t) \in Q_{T}, \\
\frac{\partial u_{p}}{\partial \vec{n}}=0, \quad(x, t) \in \partial Q_{T}, \\
u_{p}(x, 0)=f_{p}, \quad x \in \Omega,
\end{gathered}
$$

where

$$
\varphi_{p}\left(\nabla u_{p}\right)=\frac{\left|\nabla u_{p}\right|^{p+1}}{1+\left|\nabla u_{p}\right|}
$$

which implies that

$$
\varphi_{p}^{\prime}\left(\nabla u_{p}\right)=\frac{\left((p+1)+p\left|\nabla u_{p}\right|\right)\left|\nabla u_{p}\right|^{p-1} \nabla u_{p}}{\left(1+\left|\nabla u_{p}\right|\right)^{2}}
$$

Theorem 7. The approximate evolution equation (32)(34) with (35) and (36) has a weak solution $u_{p} \in L^{\infty}(0, T$; $\left.W^{1, p}(\Omega)\right), \partial u_{p} / \partial t \in L^{2}\left(Q_{T}\right)$ such that

$$
\begin{gathered}
\iint_{Q_{T}}\left[\phi \frac{\partial u_{p}}{\partial t}+\varphi_{p}^{\prime}\left(\nabla u_{p}\right) \cdot \nabla \phi\right] d x d t \\
=-\lambda \iint_{Q_{T}}\left(u_{p}-f_{p}\right) \phi d x d t, \\
\lim _{t \rightarrow 0^{+}}\left\|u_{p}(x, t)-f_{p}\right\|_{L^{2}(\Omega)}=0,
\end{gathered}
$$

where $1<p \leq 2$, for any $t \in[0, T]$, for every $\phi \in$ $L^{2}\left(0, T ; W^{1, p}(\Omega)\right), \partial \phi / \partial \vec{n}=0$, a.e. on $[0, T]$, and that $\left\|u_{p}\right\|_{L^{\infty}\left(0, T ; W^{1, p}(\Omega)\right)}+\left\|u_{p}\right\|_{L^{\infty}\left(0, T ; L^{2}(\Omega)\right)}+\left\|\partial u_{p} / \partial t\right\|_{L^{2}\left(Q_{T}\right)} \leq C$. 
Proof. We apply Rothe's method in [29] to construct an approximating solution sequence $u_{p}^{n}$ for the approximate evolution problem (32)-(34). We divide the interval $[0, T]$ into $n$ equal parts, where $h=T / n$. For any $k: 1 \leq k \leq n$, for any integer $n>0$ and a function $u(x, t)$, we have

$$
u_{p}^{n, k}(x)=u_{p}(x, k h)
$$

for $k=0,1, \ldots, n$.

We then consider the difference approximating equation of (32) as follows:

$$
\frac{u_{p}^{n, k}-u_{p}^{n, k-1}}{h}=\operatorname{div}\left(\varphi_{p}^{\prime}\left(\nabla u_{p}^{n, k}\right)\right)-\lambda\left(u_{p}^{n, k}-f_{p}\right) .
$$

Denoting $v_{p}:=u_{p}^{n, k}$, then the above equation becomes

$$
\operatorname{div}\left(\varphi^{\prime}\left(\nabla v_{p}\right)\right)-\left(\frac{1}{h}+\lambda\right) v_{p}+\left(\lambda f_{p}+\frac{u^{n, k-1}}{h}\right)=0 .
$$

The idea here is to prove that if the value of $u_{p}^{n, k-1}$ is known and $u_{p}^{n, 0}=f_{p}$, then (41) admits a weak solution $v_{p}:=u_{p}^{n, k}$.

From (41), we may back-project to the general functional $J$ defined in $H^{1}(\Omega)$, given by

$$
\begin{aligned}
J\left(v_{p}\right)= & \int_{\Omega}\left(\frac{\left|\nabla v_{p}\right|^{p+1}}{1+\left|\nabla v_{p}\right|}\right) d x+\frac{1}{2} \int_{\Omega}\left(\frac{1}{h}+\lambda\right) v_{p}^{2} d x \\
& -\int_{\Omega}\left(\lambda f_{p}+\frac{u^{n, k-1}}{h}\right) v_{p} d x .
\end{aligned}
$$

It can be shown that $J(\cdot)$ above is convex and lower semicontinuous in $W^{1, p}(\Omega)$. Hence, there exists a minimizing sequence $\left\{u_{p}^{n}\right\}$ for $J\left(v_{p}\right)$ such that $J\left(u_{p}^{n}\right)=\inf _{v_{p} \in W^{1, p}} J\left(v_{p}\right)$. For simplicity and without loss of generality, we will let $\lambda=1$. Then, for any test function $\phi(x) \in C_{0}^{\infty}(\Omega)$ with (40) and integration by parts, we have

$$
\begin{gathered}
\frac{1}{h} \int_{\Omega}\left(u_{p}^{n, k}-u_{p}^{n, k-1}\right) \phi(x) d x+\int_{\Omega} \varphi_{p}^{\prime}\left(\nabla u_{p}^{n, k}\right) \cdot \nabla \phi(x) d x \\
+\int_{\Omega}\left(u_{p}^{n, k}-f_{p}\right) \phi(x) d x=0 .
\end{gathered}
$$

To obtain the approximate solution to the whole domain $Q_{T}$, we denote

$$
\begin{aligned}
& u_{p}^{n}(x, t)=\sum_{k=1}^{n} \chi^{n, k}(t) u_{p}^{n, k} ; \quad u_{p}^{n}(x, 0)=f_{p}(x), \\
& v_{p}^{n}(x, t)=\sum_{k=1}^{n} \chi^{n, k}(t) \\
& \quad \times\left(u_{p}^{n, k-1}(x)+\lambda^{n, k}(t)\left(u_{p}^{n, k}(x)-u_{p}^{n, k-1}(x)\right)\right),
\end{aligned}
$$

where $\chi^{n, k}(t)$ is the indicator function of $t \in[(k-1) h, k h]$,

$$
\lambda^{n, k}(t)= \begin{cases}\frac{t}{h}-(k-1), & t \in[(k-1) h, k h), \\ 0, & \text { otherwise. }\end{cases}
$$

Equation (43) implies that

$$
\begin{gathered}
\iint_{Q_{T}}\left[\phi(x, t) \frac{\partial v_{p}^{n}}{\partial t}+\varphi_{p}^{\prime}\left(\nabla u_{p}^{n}\right) \cdot \nabla \phi(x, t)\right] d x d t \\
+\iint_{Q_{T}}\left(u_{p}^{n}-f_{p}\right) \phi(x, t) d x d t=0
\end{gathered}
$$

for $\phi \in C_{0}^{\infty}\left(Q_{T}\right)$.

In the steps that follow, we obtain some estimates for $u_{p}^{n}(x, t)$ and $v_{p}^{n}(x, t)$. For this purpose, let us choose the test function $\phi(x)$ in (43) such that $\phi(x)=u_{p}^{n, k}-u_{p}^{n, k-1}$. We then obtain

$$
\begin{aligned}
\frac{1}{h} \int_{\Omega} & \left(u_{p}^{n, k}-u_{p}^{n, k-1}\right)^{2} d x \\
& +\int_{\Omega} \varphi^{\prime}\left(\nabla u_{p}^{n, k}\right) \cdot\left(\nabla u_{p}^{n, k}-\nabla u_{p}^{n, k-1}\right) d x \\
& +\int_{\Omega}\left(u_{p}^{n, k}-f_{p}\right)\left(u_{p}^{n, k}-u_{p}^{n, k-1}\right) d x=0 .
\end{aligned}
$$

Applying convexity leads to

$$
\begin{aligned}
& \frac{1}{h} \int_{\Omega}\left(u_{p}^{n, k}-u_{p}^{n, k-1}\right)^{2} d x \\
& \quad+\frac{1}{2} \int_{\Omega}\left(u^{n, k}-f_{p}\right)^{2} d x+\int_{\Omega} \varphi_{p}\left(\nabla u_{p}^{n, k}\right) d x \\
& \quad \leq \int_{\Omega} \varphi_{p}\left(\nabla u_{p}^{n, k-1}\right) d x+\frac{1}{2} \int_{\Omega}\left(u_{p}^{n, k-1}-f_{p}\right)^{2} d x .
\end{aligned}
$$

Summing (48) from $k$ to $r$ for $1 \leq k \leq r \leq n$ yields

$$
\int_{\Omega} \varphi_{p}\left(\nabla u_{p}^{n, r}\right) d x \leq \int_{\Omega} \varphi_{p}\left(\nabla f_{p}\right) d x,
$$

which implies that

$$
\begin{array}{r}
\sup _{0<t<T} \int_{\Omega} \varphi_{p}\left(\nabla u_{p}^{n}\right) d x \leq \int_{\Omega} \varphi_{p}\left(\nabla f_{p}\right) d x=C_{1} \\
\left(C_{1} \equiv \text { constant }\right) .
\end{array}
$$

In addition, let us consider that

$$
\begin{aligned}
C_{1} & \geq \int_{\Omega} \varphi_{p}\left(\nabla u_{p}^{n}\right) d x=\int_{\Omega} \frac{\left|\nabla u_{p}^{n}\right|^{p+1}}{1+\left|\nabla u_{p}^{n}\right|} d x \\
& =\int_{\mid \nabla u_{p}^{n} \leq \leq k} \frac{\left|\nabla u_{p}^{n}\right|^{p+1}}{1+\left|\nabla u_{p}^{n}\right|} d x+\int_{\left|\nabla u_{p}^{n}\right|>k} \frac{\left|\nabla u_{p}^{n}\right|^{p+1}}{1+\left|\nabla u_{p}^{n}\right|} d x \\
& \geq \int_{\mid \nabla u_{p}^{n} \leq \leq 1}|\nabla u|^{p+1} d x+\int_{\left|\nabla u_{p}^{n}\right|>1} \frac{1}{2}\left|\nabla u_{p}^{n}\right|^{p},
\end{aligned}
$$

for some appropriately defined constant $k$. Observe from the above inequality that

$$
\begin{aligned}
& \int_{\left|\nabla u_{p}^{n}\right| \leq 1}\left|\nabla u_{p}^{n}\right|^{p+1} d x \leq C_{1}, \\
& \frac{1}{2} \int_{\left|\nabla u_{p}^{n}\right|>1}\left|\nabla u_{p}^{n}\right|^{p} d x \leq C_{1} .
\end{aligned}
$$


Application of Hölder inequality yields the following inequality:

$$
\begin{aligned}
\int_{\left|\nabla u_{p}^{n}\right| \leq 1}\left|\nabla u_{p}^{n}\right|^{p} d x \\
\quad \leq\left(\int_{\left|\nabla u_{p}^{n}\right| \leq 1}\left|\nabla u_{p}^{n}\right|^{p+1} d x\right)^{p /(p+1)}\left(\int_{\left|\nabla u_{p}^{n}\right| \leq 1} 1 d x\right)^{1 /(p+1)} . \\
\quad \leq C_{1}
\end{aligned}
$$

It is then clear from (53) and (54) that

$$
\int_{\Omega}\left|\nabla u_{p}^{n}\right|^{p} d x \leq C_{1} .
$$

Hence, we can conclude from (50)-(54) that

$$
\sup _{0 \leq t \leq T} \int_{\Omega}\left|\nabla u_{p}^{n}\right|^{p} d x \leq C_{1} \text {. }
$$

Now, summing (48) from $k$ to $n$ leads to

$$
\begin{aligned}
& \frac{1}{h} \sum_{k=1}^{n} \int_{\Omega}\left(u_{p}^{n, k}-u_{p}^{n, k-1}\right)^{2} d x+\frac{1}{2} \int_{\Omega}\left(u_{p}^{n}-f_{p}\right)^{2} d x \\
& \leq \int_{\Omega} \varphi_{p}\left(\nabla f_{p}\right) d x .
\end{aligned}
$$

Equation (57) implies that

$$
\frac{1}{h} \sum_{k=1}^{n} \int_{\Omega}\left(u_{p}^{n, k}-u_{p}^{n, k-1}\right)^{2} d x \leq \int_{\Omega} \varphi_{p}\left(\nabla f_{p}\right) d x=C_{1} .
$$

Moreover, by definition of $v_{p}^{n}(x, t)$, we see that

$$
\frac{\partial v_{p}^{n}}{\partial t}=\frac{1}{h} \sum_{k=1}^{n} \chi^{n, k}(t)\left(u_{p}^{n, k}-u_{p}^{n, k-1}\right)
$$

which by (58) implies that

$$
\left\|\frac{\partial v_{p}^{n}}{\partial t}\right\|_{L^{2}\left(Q_{T}\right)}^{2}=\frac{1}{h^{2}} \sum_{k=1}^{n} h\left\|u_{p}^{n, k}-u_{p}^{n, k-1}\right\|^{2} \leq C_{1} .
$$

It can be deduced from (50) that

$$
\sup _{0<t<T} \int_{\Omega} \varphi_{p}\left(\nabla v_{p}^{n}\right) d x \leq C_{1} .
$$

Now, observe from (57) that

$$
\frac{1}{2} \int_{\Omega}\left(u_{p}^{n}-f_{p}\right)^{2} d x \leq C_{1}
$$

Using Minkowski's inequality on the strength of (62), we deduce that

$$
\left\|u_{p}^{n}\right\|_{L^{2}(\Omega)}-\left\|f_{p}\right\|_{L^{2}(\Omega)} \leq\left\|u_{p}^{n}-f_{p}\right\|_{L^{2}(\Omega)} \leq C_{1}
$$

which leads to

$$
\left\|u_{p}^{n}\right\|_{L^{2}(\Omega)} \leq C_{1}+\left\|f_{p}\right\|_{L^{2}(\Omega)}=C_{2} .
$$

From the above estimate, it can be deduced that

$$
\sup _{0<t<T}\left\|v_{p}^{n}\right\|_{L^{2}(\Omega)} \leq C_{2} \text {. }
$$

Denoting $\varphi_{p}^{\prime}\left(\nabla u_{p}^{n}\right):=B u_{p}^{n}$, then from (56), (60), (61), (64), and (65), it can be seen that the sequences $\left\{u_{p}^{n}\right\},\left\{v_{p}^{n}\right\},\left\{\partial v_{p}^{n} / \partial t\right\}$, and $\left\{B u_{p}^{n}\right\}$ are bounded. Hence, there exist subsequences of $\left\{u_{p}^{n}\right\},\left\{v_{p}^{n}\right\},\left\{\partial v_{p}^{n} / \partial t\right\}$, and $\left\{B u_{p}^{n}\right\}$, respectively, denoted by the same sequences such that, as $n \rightarrow \infty$,

$$
\begin{gathered}
u_{p}^{n} \rightarrow u_{p}, \quad \text { weakly in } L^{\infty}\left(0, T ; L^{2}(\Omega)\right), \\
u_{p}^{n} \rightarrow v_{p}, \quad \text { weakly in } L^{\infty}\left(0, T ; L^{2}(\Omega)\right), \\
u_{p}^{p} \rightarrow u_{p}, \quad \text { weakly in } L^{\infty}\left(0, T ; W^{1, p}(\Omega)\right), \\
\frac{\partial v_{p}^{n}}{\partial t} \rightarrow \frac{\partial v_{p}}{\partial t}, \quad \text { weakly in } L^{2}\left(Q_{T}\right),
\end{gathered}
$$$$
B u_{p}^{n} \rightarrow Z, \quad \text { weak in } L^{\infty}\left(0, T ; L^{q}(\Omega)\right)^{N} \text {, for } q=\frac{p}{p-1},
$$

for some $u_{p}, v_{p}$, and $Z$.

Next, we show that $v_{p}=u_{p}$. Observe from (44)-(45) that

$$
v_{p}^{n}-u_{p}^{n}=\sum_{k=1}^{n} \chi^{n, k}\left(1-\lambda^{n, k}\right)\left(u_{p}^{n, k-1}-u_{p}^{n, k}\right),
$$

which by (58) leads to

$$
\left\|v_{p}^{n}-u_{p}^{n}\right\|_{L^{2}\left(\mathrm{Q}_{T}\right)}^{2} \leq \sum_{k=1}^{n} h\left\|u_{p}^{n, k}-u_{p}^{n, k-1}\right\|_{L^{2}(\Omega)}^{2} \leq C_{1} h^{2} .
$$

This implies that $\left\|v_{p}^{n}-u_{p}^{n}\right\|_{L^{2}\left(Q_{T}\right)}^{2} \leq 0$ as $h \rightarrow 0$. Since from the subsequences above it can be seen that $u_{p}^{n}$ converges to $u_{p}$ and $v_{p}^{n}$ converges to $v_{p}$, both in $L^{2}\left(Q_{T}\right)$, it follows from (68) that $v_{p}=u_{p}$.

Furthermore, from (56), (60), (64), and (65) and letting $n \rightarrow \infty$, it can be deduced that

$$
\begin{array}{r}
\left\|\frac{\partial v_{p}}{\partial t}\right\|_{L^{2}\left(Q_{T}\right)}+\left\|u_{p}\right\|_{L^{\infty}\left(0, T ; L^{2}(\Omega)\right)}+\left\|u_{p}\right\|_{L^{\infty}\left(0, T ; W^{1, p}(\Omega)\right)} \leq C_{3}, \\
\text { where } C_{3}=\left(2 C_{1}+C_{2}\right) .
\end{array}
$$

Thus, the above convergence results imply that, as $n \rightarrow \infty$ and for any $\phi \in C_{0}^{\infty}\left(Q_{T}\right)$, (46) yields

$$
\begin{aligned}
& \iint_{Q_{T}} \frac{\partial u_{p}}{\partial t} \phi d x d t \\
& \quad+\iint_{Q_{T}} Z \cdot \nabla \phi d x d t+\iint_{Q_{T}}\left(u_{p}-f_{p}\right) \phi d x d t=0 .
\end{aligned}
$$

Now, it remains to show that $Z=B u_{p}$. Here, we follow the works in $[25,30,31]$. 
Recalling that $\varphi_{p}^{\prime}\left(\nabla u_{p}^{n, k}\right):=B u_{p}^{n, k}$, then for any $w \in$ $L^{p}\left(0, T ; W^{1, p}(\Omega)\right)$ and for $k$ from 1 to $n$, by the monotonicity condition, we obtain the inequality

$$
\int_{\Omega}\left(B u_{p}^{n, k}-B w(t)\right)\left(\nabla u_{p}^{n, k}-\nabla w(t)\right) d x \geq 0
$$

Now, letting $\phi=u_{p}^{n, k}$ in (43), we obtain

$$
\begin{aligned}
\frac{1}{h} \int_{\Omega} & \left(u_{p}^{n, k}-u_{p}^{n, k-1}\right) u_{p}^{n, k} d x \\
& +\int_{\Omega} B u_{p}^{n, k} \cdot \nabla u_{p}^{n, k} d x+\int_{\Omega}\left(u_{p}^{n, k}-f_{p}\right) u_{p}^{n, k} d x=0 .
\end{aligned}
$$

Applying Young's inequality on the first term of (72) together with the inequality $(71)$ and integrating over $((k-1) h, k h)$, we obtain

$$
\begin{aligned}
\frac{1}{2} \int_{\Omega} & {\left[\left|u_{p}^{n, k}\right|^{2}-\left|u_{p}^{n, k-1}\right|^{2}\right] d x } \\
& +\int_{(k-1) h}^{k h} \int_{\Omega} B u_{p}^{n, k} \cdot \nabla w d x d t \\
& +\int_{(k-1) h}^{k h} \int_{\Omega} B w\left(\nabla u_{p}^{n, k}-\nabla w\right) d x d t \\
& +h \int_{\Omega}\left(u_{p}^{n, k}-f_{p}\right) u_{p}^{n, k} d x \leq 0 .
\end{aligned}
$$

Summing up (73) for $k$ from 1 to $n$, we obtain

$$
\begin{aligned}
\frac{1}{2} \int_{\Omega} & {\left[\left|u_{p}^{n}(T)\right|^{2}-\left|f_{p}\right|^{2}\right] d x+\iint_{Q_{T}} B u_{p}^{n} \cdot \nabla w d x d t } \\
& +\iint_{Q_{T}} B w\left(\nabla u_{p}^{n}-\nabla w\right) d x d t \\
& +\iint_{Q_{T}}\left(u_{p}^{n}-f_{p}\right) u_{p}^{n} d x d t \leq 0 .
\end{aligned}
$$

Recalling the convergence sets in (66) and letting $n \rightarrow \infty$, (74) yields

$$
\begin{gathered}
\frac{1}{2} \int_{\Omega}\left[\left|u_{p}(T)\right|^{2}-\left|f_{p}\right|^{2}\right] d x+\iint_{\mathrm{Q}_{T}} Z \cdot \nabla w d x d t \\
\quad+\iint_{\mathrm{Q}_{T}} B w\left(\nabla u_{p}-\nabla w\right) d x d t \\
\quad+\iint_{Q_{T}}\left(u_{p}-f\right) u_{p} d x d t \leq 0 .
\end{gathered}
$$

Equation (75) may be rewritten in the form

$$
\begin{gathered}
\iint_{\mathrm{Q}_{T}} u_{p} \frac{\partial u_{p}}{\partial t} d x d t+\iint_{Q_{T}} Z \cdot \nabla w d x d t \\
+\iint_{Q_{T}} B w\left(\nabla u_{p}-\nabla w\right) d x d t \\
+\iint_{Q_{T}}\left(u_{p}-f_{p}\right) u_{p} d x d t \leq 0 .
\end{gathered}
$$

Now, setting $\phi=u_{p}$ in (70), we obtain

$$
\begin{gathered}
\iint_{Q_{T}} u_{p} \frac{\partial u_{p}}{\partial t} d x d t+\iint_{Q_{T}} Z \cdot \nabla u_{p} d x d t \\
+\iint_{Q_{T}}\left(u_{p}-f_{p}\right) u_{p} d x d t=0 .
\end{gathered}
$$

Then, substituting equation (77) into (76) leads to

$$
\iint_{Q_{T}}(Z-B w)\left(\nabla u_{p}-\nabla w\right) d x d t \geq 0 .
$$

Since $w$ is arbitrary, we may set $w=u_{p}-\kappa s$ where $\kappa>0$ and $\nabla s \in L^{\infty}\left(0, T ; W^{1, p}(\Omega)\right)$. We then have

$$
\iint_{Q_{T}}\left(Z-B\left(u_{p}-\kappa s\right)\right) \nabla s d x d t \geq 0
$$

Sending $\kappa \rightarrow 0$, we obtain

$$
\iint_{Q_{T}}\left(Z-B u_{p}\right) \nabla s d x d t \geq 0, \quad \forall s \in L^{\infty}\left(0, T ; W^{1, p}(\Omega)\right) .
$$

Observe that, in fact, equality holds if we set $s=-s$ in the inequality above. We thus deduce that $B u_{p}=Z$. Hence, $\varphi_{p}^{\prime}\left(\nabla u_{p}\right)=B\left(u_{p}\right)=Z$ a.e. in $Q_{T}$. Therefore, (70) together with (80) leads to the identity (37). Thus, $u_{p}$ is a weak solution to (32)-(34), taken together with (35) and (36).

To prove the second part, that is, the relation (38), we let in (70) $\phi=u_{p}(x, t)$ and then $\phi=u_{p}\left(x, t_{1}\right)$, for $0 \geq t_{1} \geq t_{2} \geq T$. Consider

$$
\begin{aligned}
\int_{\Omega}\left(u_{p}^{2}\left(x, t_{2}\right)-u_{p}^{2}\left(x, t_{1}\right)\right) d x \\
=-2 \int_{t_{1}}^{t_{2}} \int_{\Omega} \varphi_{p}^{\prime}\left(\nabla u_{p}(x, t)\right) \nabla u_{p}(x, t) d x d t \\
\quad-2 \int_{t_{1}}^{t_{2}} \int_{\Omega}\left(u_{p}(x, t)-f_{p}\right) u_{p}(x, t) d x d t \\
\int_{\Omega}\left(u_{p}\left(x, t_{1}\right) u_{p}\left(x, t_{2}\right)-u_{p}^{2}\left(x, t_{1}\right)\right) d x \\
=-\int_{t_{1}}^{t_{2}} \int_{\Omega} \varphi_{p}^{\prime}\left(\nabla u_{p}(x, t)\right) \nabla u_{p}\left(x, t_{1}\right) d x d t \\
\quad-\int_{t_{1}}^{t_{2}} \int_{\Omega}\left(u_{p}(x, t)-f_{p}\right) u_{p}\left(x, t_{1}\right) d x d t .
\end{aligned}
$$

Observe that from the above equation we have

$$
\begin{aligned}
\int_{\Omega}\left|u_{p}\left(x, t_{2}\right)-u_{p}\left(x, t_{1}\right)\right|^{2} d x \\
=\int_{\Omega}\left(u_{p}^{2}\left(x, t_{2}\right)-u_{p}^{2}\left(x, t_{1}\right)\right) d x \\
\quad+2 \int_{\Omega}\left(u_{p}^{2}\left(x, t_{1}\right)-u_{p}\left(x, t_{1}\right) u_{p}\left(x, t_{2}\right)\right) d x
\end{aligned}
$$




$$
\begin{aligned}
= & -2 \int_{t_{1}}^{t_{2}} \int_{\Omega} \varphi_{p}^{\prime}\left(\nabla u_{p}(x, t)\right) \nabla u_{p}(x, t) d x d t \\
& -2 \int_{t_{1}}^{t_{2}} \int_{\Omega}\left(u_{p}(x, t)-f_{p}\right) u_{p}(x, t) d x d t \\
& +2 \int_{t_{1}}^{t_{2}} \int_{\Omega} \varphi_{p}^{\prime}\left(\nabla u_{p}(x, t)\right) \nabla u_{p}\left(x, t_{1}\right) d x d t \\
& +2 \int_{t_{1}}^{t_{2}} \int_{\Omega}\left(u_{p}(x, t)-f_{p}\right) u_{p}\left(x, t_{1}\right) d x d t .
\end{aligned}
$$

We deduce from the above equation that

$$
\lim _{t \rightarrow 0^{+}}\left\|u_{p}(x, t)-f_{p}\right\|_{L^{2}(\Omega)}=0, \quad \text { since } u_{p}(x, 0)=f_{p} .
$$

\subsection{Existence and Uniqueness of the Solution to the Evolution} Problem (27)-(29)

Theorem 8. Let $f \in B V(\Omega) \cap L^{2}(\Omega)$. Then, there exists a unique weak solution $u \in L^{\infty}\left(0, T ; B V(\Omega) \cap L^{2}(\Omega)\right)$, $\partial u / \partial t \in$ $L^{2}\left(Q_{T}\right)$, and $u(x, 0)=f$ in the trace sense.

Proof. By Theorem 7 , there exists $u_{p}$, which is a weak solution to the approximate problem (32)-(34) and a constant $C$ such that

$$
\left\|u_{p}\right\|_{L^{\infty}\left(0, T ; W^{1, p}(\Omega)\right)}+\left\|u_{p}\right\|_{L^{\infty}\left(0, T ; L^{2}(\Omega)\right)}+\left\|\frac{\partial u_{p}}{\partial t}\right\|_{L^{2}\left(Q_{T}\right)} \leq C .
$$

From (84), we deduce that there exists a subsequence of $\left\{u_{p}\right\}$ denoted by $\left\{u_{p}\right\}$ itself and a function $u \in L^{\infty}(0, T ; \mathrm{BV}(\Omega) \cap$ $L^{2}(\Omega)$ with $\partial u / \partial t \in L^{2}\left(Q_{T}\right)$ such that, as $p \rightarrow 1^{+}$,

$$
\begin{array}{r}
u_{p} \longrightarrow u \text { in } L^{1}(\Omega), \text { given }\|D u\| \leq \liminf _{p \rightarrow 1^{+}}\left\|D u_{p}\right\|_{L^{p}(\Omega)}, \\
\text { a.e. } \in(0 . T) .
\end{array}
$$

From (84), we may also deduce that

$$
\frac{\partial u_{p}}{\partial t} \rightarrow \frac{\partial u}{\partial t} \quad \text { weakly in } L^{2}\left(Q_{T}\right)
$$

Since $\operatorname{BV}(\Omega) \hookrightarrow L^{p}(\Omega),(1 \leq p \leq N /(N-1))$ continuously $[7,27,31,32]$ and also considering $(84)$, we deduce that

$$
u_{p} \longrightarrow u, \quad \text { strongly in } L^{2}\left(Q_{T}\right) .
$$

Applying the method in [25], we next show that $\varphi_{p}^{\prime}\left(\nabla u_{p}\right)$ is weakly compact in $L^{1}\left(Q_{T} ; \mathbb{R}^{N}\right)$. Using Jensen's inequality and Hölder's inequality, we may have that

$$
\begin{aligned}
\left|\iint_{Q_{T}} \varphi_{p}^{\prime}\left(\nabla u_{p}\right) d x d t\right| & \leq \iint_{Q_{T}}\left|\varphi_{p}^{\prime}\left(\nabla u_{p}\right)\right| d x d t \\
& \leq p \iint_{Q_{T}}\left|\nabla u_{p}\right|^{p-1} d x d t
\end{aligned}
$$

$$
\begin{aligned}
& \leq p\left(\iint_{Q_{T}}\left|\nabla u_{p}\right|^{p} d x d t\right)^{(p-1) / p}(1)^{1 / p} \\
& \leq p(C)^{(p-1) / p} \mathscr{L}^{N}\left(Q_{T}\right)^{1 / p} .
\end{aligned}
$$

Hence, $\varphi_{p}^{\prime}\left(\nabla u_{p}\right)$ is bounded and equi-integrable in $L^{1}\left(Q_{T} ; \mathbb{R}^{N}\right)$ and is therefore weakly compact in $L^{1}\left(Q_{T} ; \mathbb{R}^{N}\right)$. Thus, we may deduce that

$$
\varphi_{p}^{\prime}\left(\nabla u_{p}\right) \rightarrow z \quad \text { weakly in } L^{1}\left(Q_{T} ; \mathbb{R}^{N}\right)
$$

Hence, we obtain

$$
\begin{aligned}
& \iint_{Q_{T}} \frac{\partial u}{\partial t} \phi d x d t \\
& \quad+\iint_{Q_{T}} z \cdot \nabla \phi d x d t+\iint_{Q_{T}}(u-f) \phi d x d t=0
\end{aligned}
$$

for every $\phi \in C_{0}^{\infty}\left(Q_{T}\right)$ and $\partial u / \partial t=\operatorname{div}(z)-\lambda(u-f)$ in $\mathscr{D}^{\prime}\left(Q_{T}\right)$.

Now, it remains to show that $\|z\|_{L^{\infty}\left(Q_{T} ; \mathbb{R}^{N}\right)} \leq 1$.

For any $r>0$ and setting $B_{p, r}=\left\{(x, t) \in Q_{T}:\left|\nabla u_{p}\right|>r\right\}$, we have

$$
\left|B_{p, r}\right| \leq \frac{C}{r^{p}}, \quad \text { for any } p>1, r>0
$$

Then, as above, there exist a function $g_{r} \in L^{1}\left(Q_{T}: \mathbb{R}^{N}\right)$ and the indicator function $\chi_{B_{p, r}}$ of $B_{p, r}$ such that

$\varphi_{p}^{\prime}\left(\nabla u_{p}\right) \chi_{B_{p, r}} \longrightarrow g_{r} \quad$ weakly in $L^{1}\left(Q_{T} ; \mathbb{R}^{N}\right)$ as $p \longrightarrow 1^{+}$.

And, for any $\phi \in L^{\infty}\left(Q_{T} ; \mathbb{R}^{N}\right)$, it can be shown that

$$
\left|\iint_{Q_{T}} \varphi_{p}^{\prime}\left(\nabla u_{p}\right) \cdot \phi \chi_{B_{p, r}} d x d t\right| \leq \frac{C}{r} .
$$

Letting $p \rightarrow 1^{+}$, we obtain

$$
\iint_{Q_{T}}\left|g_{r}\right| d x d t \leq \frac{C}{r}, \quad \text { for any } r>0
$$

Now, taking into consideration equations (91), (93) and (94), we deduce that

$$
\left|\iint_{Q_{T}} \varphi_{p}^{\prime}\left(\nabla u_{p}\right) \cdot \phi \chi_{Q_{T} / B_{p, r}}\right| \leq r^{p-1}, \quad \text { for any } p>1,
$$

letting $p \rightarrow 1^{+}$, we get that $\varphi_{p}^{\prime}\left(\nabla u_{p}\right) \cdot \phi \chi_{Q_{T} / B_{p, r}}$ converges weakly to some function $f_{r} \in L^{1}\left(Q_{T} ; \mathbb{R}^{N}\right)$ with $\left\|f_{r}\right\|_{L^{\infty}\left(Q_{T} ; \mathbb{R}^{N}\right)} \leq 1$. And since for any $r>0$ we may write $z=f_{r}+g_{r}$, with $g_{r}$ satisfying condition (94), it follows that $\|z\|_{L^{\infty}\left(Q_{T} ; \mathbb{R}^{N}\right)} \leq 1$. 
Next, we verify the solution definition inequality (31). By setting $\phi=\left(u_{p}-v_{n}\right) \eta(t)$, (37) leads to

$$
\begin{aligned}
\iint_{Q_{T}}\left(u_{p}-v_{n}\right) \eta(t) \frac{\partial u_{p}}{\partial t} d x d t \\
=-\iint_{Q_{T}} \varphi_{p}^{\prime}\left(\nabla u_{p}\right) \cdot \nabla\left(\left(u_{p}-v_{n}\right) \eta(t)\right) d x d t \\
\quad-\lambda \iint_{Q_{T}}\left(u_{p}-f_{p}\right)\left(u_{p}-v_{n}\right) \eta(t) d x d t .
\end{aligned}
$$

Taking the limit as $p \rightarrow 1^{+}$gives the inequality

$$
\begin{aligned}
\iint_{Q_{T}} & \left(u(t)-v_{n}(t)\right) \eta(t) \frac{\partial u}{\partial t} d x d t \\
\leq & \iint_{Q_{T}} z(t) \cdot \nabla v_{n} \eta(t) d x d t-\int_{0}^{T}\|\nabla u\| \eta(t) d t \\
& -\lambda \iint_{Q_{T}}(u-f)\left(u-v_{n}\right) \eta(t) d x d t
\end{aligned}
$$

Taking the limit as $n \rightarrow \infty$ for every $v \in L^{\infty}\left(0, T ; W^{1,1}(\Omega)\right)$ and recognizing the arbitrary nature of $\eta(t)$, we obtain

$$
\begin{aligned}
& \int_{\Omega}(u(t)-v(t)) \frac{\partial u}{\partial t} d x \\
& \quad \leq \int_{\Omega} z(t) \cdot \nabla v d x-\|D u\|-\lambda \int_{\Omega}(u-f)(u-v) d x
\end{aligned}
$$

for every $v \in L^{\infty}\left(0, T ; W^{1,1}(\Omega)\right)$, a.e. in $[0, T]$. That concludes the proof of the existence of the entropy solution of problem (27)-(29).

Uniqueness of Weak Solution. Let $u_{1}$ and $u_{2}$ be two entropy solutions of the problem (27)-(29), such that, respectively, their initial data are $u_{1}(x, 0)=f_{10}$ and $u_{2}(x, 0)=f_{20}$. Then, for every $v \in L^{\infty}\left(0, T ; W^{1,1}(\Omega)\right)$, a.e. on $[0, T]$, there exists $z_{1}, z_{2} \in L^{\infty}\left(Q_{T} ; \mathbb{R}^{N}\right)$ such that

$$
\begin{aligned}
& \int_{\Omega}\left(u_{1}-v\right) \frac{\partial u_{1}}{\partial t} d x \\
& \quad \leq \int_{\Omega} z_{1} \cdot \nabla v d x-\left\|\nabla u_{1}\right\|-\lambda \int_{\Omega}\left(u_{1}-f\right)\left(u_{1}-v\right) d x \\
& \int_{\Omega}\left(u_{2}-v\right) \frac{\partial u_{2}}{\partial t} d x \\
& \quad \leq \int_{\Omega} z_{2} \cdot \nabla v d x-\left\|\nabla u_{2}\right\|-\lambda \int_{\Omega}\left(u_{2}-f\right)\left(u_{2}-v\right) d x
\end{aligned}
$$

In addition, let $u_{1 n}$ and $u_{2 n}$ be approximations, respectively, for $u_{1}$ and $u_{2}$, such that

$$
\begin{gathered}
\lim _{n \rightarrow \infty}\left(\left\|\nabla u_{1 n}\right\|_{L^{1}(\Omega)}-\left\|D u_{1}\right\|\right)=0, \\
\lim _{n \rightarrow \infty}\left(\left\|\nabla u_{2 n}\right\|_{L^{1}(\Omega)}-\left\|D u_{2}\right\|\right)=0, \\
\lim _{n \rightarrow \infty}\left\|u_{1 n}-u_{1}\right\|=0, \\
\lim _{n \rightarrow \infty}\left\|u_{2 n}-u_{2}\right\|=0,
\end{gathered}
$$

a.e. on $[0, T]$. By setting $v=u_{2 n}$ in (99) and $v=u_{1 n}$ in (100), adding the two equations, applying Lemma 3 , and rearranging the result, we obtain

$$
\begin{aligned}
& \int_{\Omega}\left(u_{1}-u_{2}\right)\left(\frac{\partial u_{1}}{\partial t}-\frac{\partial u_{2}}{\partial t}\right) d x \\
& \quad+\int_{\Omega}\left(u_{1}-u_{1 n}\right) \frac{\partial u_{2}}{\partial t} d x+\int_{\Omega}\left(u_{2}-u_{2 n}\right) \frac{\partial u_{1}}{\partial t} d x \\
& \leq \int_{\Omega} z_{1} \cdot \nabla u_{2 n} d x-\left\|\nabla u_{2}\right\|+\int_{\Omega} z_{2} \cdot \nabla u_{1 n} d x-\left\|\nabla u_{1}\right\| \\
& \quad-\frac{\lambda}{2} \int_{\Omega}\left(\left(u_{1 n}-f\right)^{2}-\left(u_{1}-f\right)^{2}\right) d x \\
& \quad-\frac{\lambda}{2} \int_{\Omega}\left(\left(u_{2 n}-f\right)^{2}-\left(u_{2}-f\right)^{2}\right) d x
\end{aligned}
$$

Then, integrating the inequality above from 0 to $t$ and taking the limit as $n \rightarrow \infty$, yield

$$
\int_{\Omega}\left(u_{1}-u_{2}\right)^{2} d x \leq\left(f_{10}-f_{20}\right)^{2}
$$

This establishes the uniqueness of the entropy solution.

\section{Numerical Experiments}

In this section, we show the performance of the proposed formulation in denoising images involving a Gaussian white noise. The results are then compared with those obtained by the classical methods of Perona and Malik (PM) [1], Rudin et al. (TV) [8], and Guo et al. [5].

The following numerical scheme has been proposed for the implementation of the model.

5.1. Additive Operator Splitting (AOS) Scheme. Here, we have implemented the evolution problem (27)-(29) using AOS scheme proposed by Weickert et al. in [33]. Thus, the equations are discretized as follows:

$$
\begin{gathered}
\lambda^{0}=0, \\
u^{n+1}=\frac{1}{m} \sum_{l=1}^{m}\left[I-m \tau A_{l}\left(u^{k}\right)\right]^{-1}\left[u^{n}+\lambda \tau\left(f-u^{n}\right)\right], \\
\operatorname{div}^{n}=\frac{\left(u^{n+1}-u^{n}\right)}{\tau}, \\
\lambda^{n}=\frac{1}{\sigma^{2} M N}(u-f) \operatorname{div}^{n}, \\
u_{i, j}^{0}=f_{i, j}=f(i h, j h), \\
u_{i, 0}^{n}=u_{i, 1}^{n}, \quad u_{0, j}^{n}=u_{1, j}^{n}, \\
u_{I, i}^{n}=u_{I-1, i}^{n}, \quad u_{i, J}^{n}=u_{i, J-1}^{n},
\end{gathered}
$$


where $A_{l}\left(u^{n}\right)=\left[a_{i, j}\left(u^{n}\right)\right]$,

$$
\begin{gathered}
a_{i, j}\left(u^{n}\right):= \begin{cases}\frac{C_{i}^{n}+C_{j}^{n}}{2 h^{2}}, & {[j \in \mathcal{N}(i)],} \\
-\sum_{N \in \mathscr{N}(i)} \frac{C_{i}^{n}+C_{N}^{n}}{2 h^{2}}, & (j=i), \\
0, & \text { else, }\end{cases} \\
C_{i}^{n}:=\frac{2+K\left|\nabla u_{i, j}^{n}\right|}{\left(1+\left|\nabla u_{i, j}^{n}\right|\right)^{2}},
\end{gathered}
$$

where

$$
\left|\nabla u_{i, j}^{n}\right|=\frac{1}{2} \sum_{p, q \in \mathcal{N}(i)} \frac{\left|u_{p}^{n}-u_{q}^{n}\right|}{2 h},
$$

where $\mathcal{N}(i)$ is the set of the two neighbors of pixel $i$ (boundary pixels have only one neighbor) and $K$ is introduced, for convenience, as a tuning parameter in the implementation of the proposed formulation.

5.2. Discussion of the Results. The experiments in this work have been performed on a Compaq610 computer, having Intel(R) Core (TM)2 Duo CPU T5870 each $2.00 \mathrm{GHz}$, physical RAM of $4.00 \mathrm{~GB}$, and Professional Windows 8 64-bit Operating System, on MATLAB R2013b. The image restoration performance has been measured in terms of the peak signal-to-noise ratio (PSNR), mean absolute deviation/error (MAE), structural similarity index measure (SSIM), the measure of similarity of edges $\left(\mathrm{PSNR}_{\mathrm{E}}\right)$, and visual effects. The iteration stopping mechanism is based on the maximal PSNR.

At the end of iteration process, the PSNR, MAE, SSIM, and $\mathrm{PSNR}_{\mathrm{E}}$ values are recorded. PSNR and MAE values as discussed in [34] are, respectively, given by the following formulas:

$$
\begin{gathered}
\operatorname{PSNR}\left(u, u_{0}\right)=10 \log _{10} \frac{M N\left|\max u_{0}-\min u_{0}\right|^{2}}{\left\|u-u_{0}\right\|_{L^{2}}^{2}} \mathrm{~dB}, \\
\operatorname{MAE}\left(u, u_{0}\right)=\frac{\left\|u-u_{0}\right\|_{L^{1}}}{M N},
\end{gathered}
$$

where $u_{0}$ denotes the noise-free image, $u$ is the denoised image, $M \times N$ is the dimension of image, and $\mid \max u_{0}$ $\min u_{0} \mid$ yields the gray scale range of the original image.

SSIM, designed by Wang et al. [35], is a quality metric used to measure the similarity between any two images. It is widely considered to work in a manner analogous to the human visual system. As opposed to the PSNR, SNR, MAE, and MSE, which are error based measures, SSIM models image distortion as a combination of loss of correlation, luminance degradation, and contrast distortion. Given any two images $u$ and $u_{0}$, SSIM measure is given by the formula

$$
\operatorname{SSIM}\left(u, u_{0}\right)=L\left(u, u_{0}\right) \cdot C\left(u, u_{0}\right) \cdot R\left(u, u_{0}\right) .
$$

$L\left(u, u_{0}\right)=\left(2 \mu_{u} \mu_{u_{0}}+k_{1}\right) /\left(\mu_{u}^{2}+\mu_{u_{0}}^{2}+k_{1}\right)$ compares the two images' mean luminance $\mu_{u}$ and $\mu_{u_{0}}$. The maximal value of
$L\left(u, u_{0}\right)=1$, if $\mu_{u}=\mu_{u_{0}}, C\left(u, u_{0}\right)=\left(2 \sigma_{u} \sigma_{u_{0}}+k_{2}\right) /\left(\sigma_{u}^{2}+\right.$ $\left.\sigma_{u_{0}}^{2}+k_{2}\right)$, measures the closeness of contrast of the two images $u$ and $u_{0}$. Contrast is determined in terms of standard deviation, $\sigma$. Contrast comparison measure $C\left(u, u_{0}\right)=1$ maximally if and only if $\sigma_{u}=\sigma_{u_{0}}$, that is, when the images have equal contrast.

$R\left(u, u_{0}\right)=\left(\sigma_{u u_{0}}+k_{3}\right) /\left(\sigma_{u} \sigma_{u_{0}}+k_{3}\right)$, where $\sigma_{u u_{0}}$ is covariance between $u$ and $u_{0}$, is a structure comparison measure which determines the correlation between the images $u$ and $u_{0}$. It attains maximal value of 1 if structurally the two images coincide, but its value is equal to zero when there is absolutely no structural coincidence. The quantities $k_{1}, k_{2}$, and $k_{3}$ are small positive constants that avert the possibility of having zero denominators.

Taking the edge map $\operatorname{EM}(u)$ from (27) with $K$ the tuning parameter, we have

$$
\operatorname{EM}(u)=\frac{2+K|\nabla u|}{(1+|\nabla u|)^{2}} .
$$

Hence, the corresponding edge similarity measure is given by

$$
\operatorname{PSNR}_{\mathrm{E}}=\operatorname{PSNR}\left(\operatorname{EM}(u), \operatorname{EM}\left(u_{0}\right)\right) \mathrm{dB} \quad[5] .
$$

All these measures have been deployed not only to judge the extent of signal recovery, but also to see the extent of structural coincidence between the original image and the reconstruction image. It should be noted, however, that there is no hard and fast rule for selecting between the error based measures and the structural similarity index measure [36]. Therefore, any or both can be used at the same time to measure the quality of image recovery.

We have compared results of the construction by our method to those of Perona-Malik method [1], total variation method of Rudin et al. [8], and the D- $\alpha$-PM algorithm proposed by [5]. Adjustable parameters in the experiments were time step $\tau$, thresholding $\backslash$ tuning parameter $K$, and others depending on the respective model. However, fidelity parameter $\lambda$ was dynamically obtained in the course and process of iteration.

In Tables 1 and 2, we have, respectively, presented the results of the reconstruction for the synthetic image, Figure 1, and those of the textured image, Lena, Figure 2. The comparisons are based upon PSNR, MAE, SSIM, and $\mathrm{PSNR}_{\mathrm{E}}$ values and visual effects.

For the nontextured synthetic image, Figure 1, it is observed that, in spite of higher CPU time, our method enjoys superior signal and structural recovery, at PSNR $=38.52$, MAE $=3.16$, and SSIM $=0.9905$ (see Table 1). In addition, as evidenced by Figure 1(e), reconstructions due the proposed model do not show the relative blurriness speckles evident in Figure 1(c) (by PM method); it does not manifest the relative blockiness visible on Figure 1(d) (by TV method) neither does it show heavily jagged edges apparent in Figure 1(f) due to the D- $\alpha$-PM algorithm. Comparing Figure 1(e) produced by our method and Figure 1(a) of the original image, it can be observed that the differences are extremely marginal. The edges also seem better preserved and sharper.

Furthermore, although in terms of numerical metrics our method compares fairly well with those obtained by the 
TABLE 1: Numerical results for synthetic image $(300 \times 300)$ experiments.

\begin{tabular}{|c|c|c|c|c|c|c|c|c|c|}
\hline \multirow{2}{*}{ Algorithm } & \multicolumn{3}{|c|}{ Parameters } & \multirow{2}{*}{ Number of steps } & \multirow{2}{*}{ CPU time (sec) } & \multirow{2}{*}{ PSNR } & \multirow{2}{*}{ MAE } & \multirow{2}{*}{ SSIM } & \multirow{2}{*}{$\mathrm{PSNR}_{\mathrm{E}}$} \\
\hline & $\sigma$ & $K$ & $\tau$ & & & & & & \\
\hline PM & 30 & 4 & 0.25 & 356 & 1.56 & 36.57 & 3.78 & 0.9817 & 25.06 \\
\hline TV & 30 & & 0.25 & 205 & 1.66 & 34.56 & 4.73 & 0.9725 & 14.75 \\
\hline $\mathrm{D}-\alpha-\mathrm{PM}$ & 30 & 1 & 0.25 & 40 & 2.33 & 37.2 & 3.18 & 0.9825 & 24.7 \\
\hline Our method & 30 & 0.01 & 5 & 65 & 3.98 & 38.52 & 3.16 & 0.9905 & 24.90 \\
\hline
\end{tabular}

TABLE 2: Numerical results for Lena image $(300 \times 300)$ experiments.

\begin{tabular}{|c|c|c|c|c|c|c|c|c|c|}
\hline \multirow{2}{*}{ Algorithm } & \multicolumn{3}{|c|}{ Parameters } & \multirow{2}{*}{ Number of steps } & \multirow{2}{*}{ CPU time (sec) } & \multirow{2}{*}{ PSNR } & \multirow{2}{*}{ MAE } & \multirow{2}{*}{ SSIM } & \multirow{2}{*}{$\mathrm{PSNR}_{\mathrm{E}}$} \\
\hline & $\sigma$ & $K$ & $\tau$ & & & & & & \\
\hline $\mathrm{PM}$ & 30 & 5 & 0.25 & 113 & 0.45 & 25.94 & 12.88 & 0.6939 & 20.69 \\
\hline TV & 30 & & 0.25 & 129 & 1.15 & 27.75 & 10.45 & 0.7698 & 20.70 \\
\hline $\mathrm{D}-\alpha-\mathrm{PM}$ & 30 & 4 & 2 & 10 & 0.50 & 28.15 & 7.21 & 0.7780 & 24.57 \\
\hline Our method & 30 & 0.1 & 5 & 15 & 1.07 & 27.84 & 10.20 & 0.7805 & 26.34 \\
\hline
\end{tabular}

D- $\alpha$-PM, it should be noted that the increased number of parameters in the D- $\alpha$ that have to be manually tweaked or manipulated to obtain optimal results aggregately hinders the effectiveness of the method.

For the textured image of Lena in Figure 2, the metrics evidence that our method still gives better signal recovery and structural coincidence compared to its comparisons (see Table 2). By visual observation, Figure 2(c) produced by PM method shows speckles and blur, while Figure 2(d) shows heavy presence of staircase effect, and Figure 2(f) shows heavier ramp-like features. However, Figure 2(e) produced by our method not only shows no speckles and blur but also manifests significant reduction of staircase effect.

In respect of the edge similarity measure $\mathrm{PSNR}_{E}$, for the nontexture image (see Figure 1), we observe a phenomenon where the PM method only marginally beats the proposed method. However, the method far outperforms the TV method. For the texture image (see Figure 2), experiments show that the proposed method generally outperforms the $\mathrm{PM}, \mathrm{TV}$, and D- $\alpha$-PM methods in reconstruction of image from noise (see Tables 1 and 2).

In addition, experiments demonstrate that the tuning parameter $K$ introduced in the implementation of the proposed model is texture sensitive. In the nontextured images, $K$ tends to be very small, while as texture increases the value of $K$ also tends to increase. Generally observed is that the AOS implementation algorithm shows gray value invariance, stability based on extremum principle, Lyapunov functionals, and convergence even for larger values of the scale parameter $\tau$ [33]. This does not apply in the case of TV and PM, since they become unstable for $\tau>0.25$. The D- $\alpha$-PM survives this eventuality only when implemented by the AOS algorithm.

Generally, therefore, the proposed formulation performs better than TV method and PM method and indeed fairly better than the D- $\alpha$-PM algorithm not only in the quantitative metric measures but also in terms of visual effect. The D- $\alpha$-PM method competes fairly well with the proposed method. However, the increased number of parameters in the D- $\alpha$-PM may disadvantage its practical ability to produce optimal results. Moreover, our formulation seems to handle texture images better than its comparison. Finally, given the nonmonotone nature of the flux function of the D- $\alpha$-PM model proposed by Guo et al. [5], it is an extremely ill-posed problem, generating both backward and forward diffusion at various times in the evolution process. Therefore, analytically speaking, its ability to arrive at steady state and produce data with minimum energy is highly in mathematical doubt. Notwithstanding the foregoing, the discrete or numerical implementation of the D- $\alpha$-PM method still does give an approximated solution.

\section{Conclusion}

In this paper, we have proposed an alternative framework for total variation based image denoising models. The model is based on minimization of total variation with a functional coefficient. We set out to determine factors to consider when choosing the functional coefficient for the minimization potential, and we have established that, for a well-behaved minimization functional, it is desirable that the functional coefficient be of sublinear growth at infinity. The chosen functional coefficient, apart from its sublinear growth at infinity, must be one that results in a potential which is nondecreasing, of linear growth at infinity, and convex. Convexity can then be used to obtain lower semicontinuity, as it is also instrumental in determination of existence of a minimizer to the functional.

We picked on an example to illustrate the effectiveness of the consideration. We have shown for the example that existence and uniqueness of minimizer can be established and that the evolution equation has a unique entropy solution. In addition, numerical implementation of the evolution equation on images demonstrates better denoising results than those offered by traditional competition due to $\mathrm{PM}$ method, TV method, and even the D- $\alpha$-PM in [5].

The successful application or otherwise of any regularization formulation in practical denoising always depends on the type of image under consideration, the type of noise involved, the application intended for the restored image, and indeed the platform upon which the formulation is implemented. 


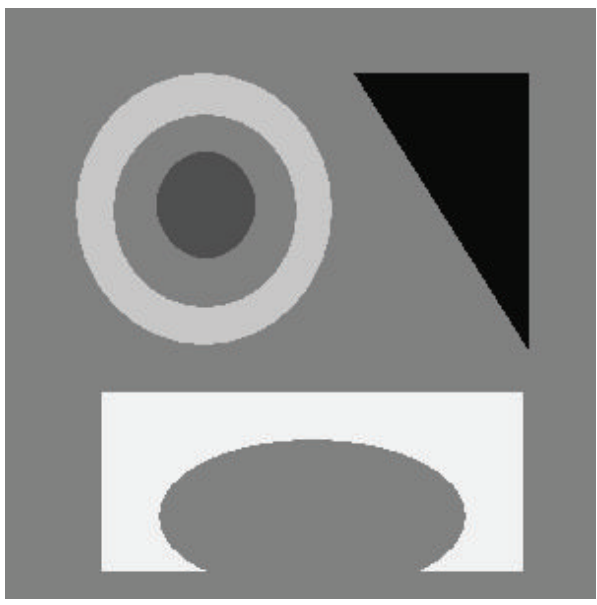

(a)

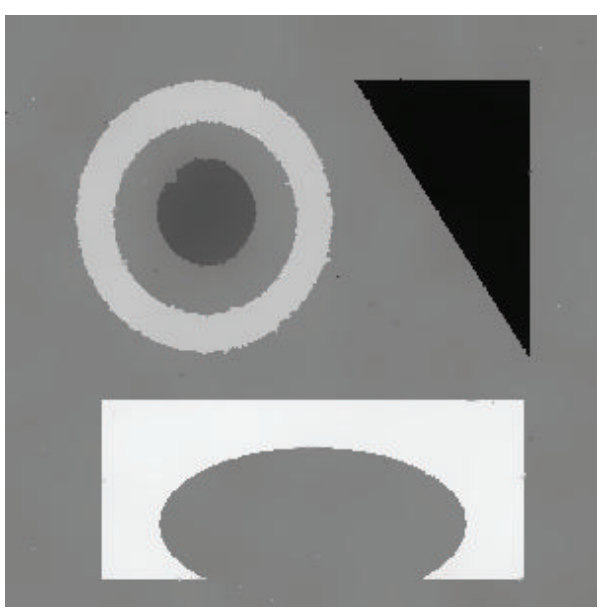

(c)

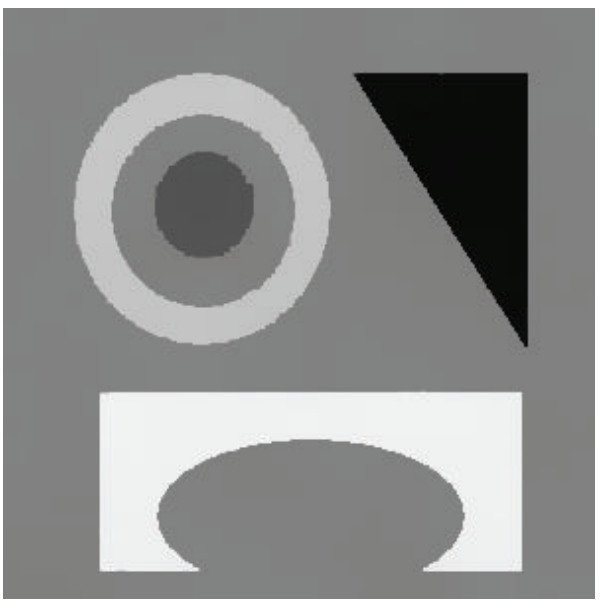

(e)

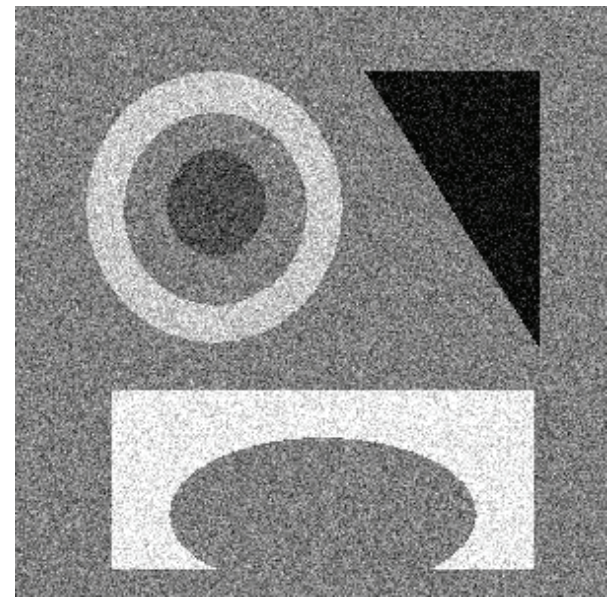

(b)

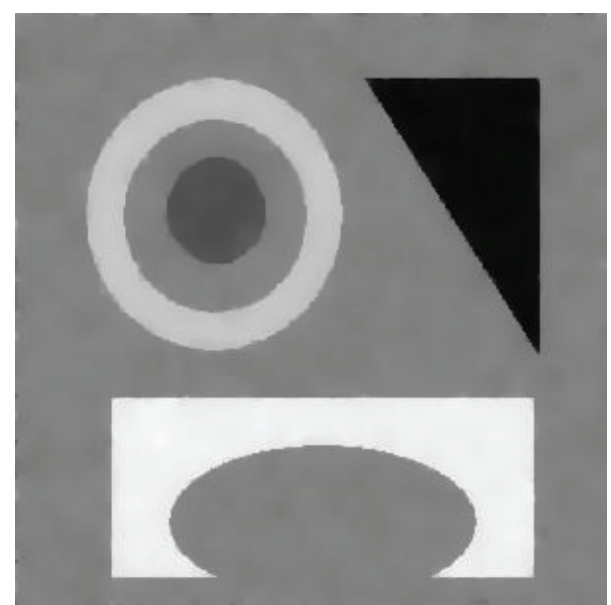

(d)

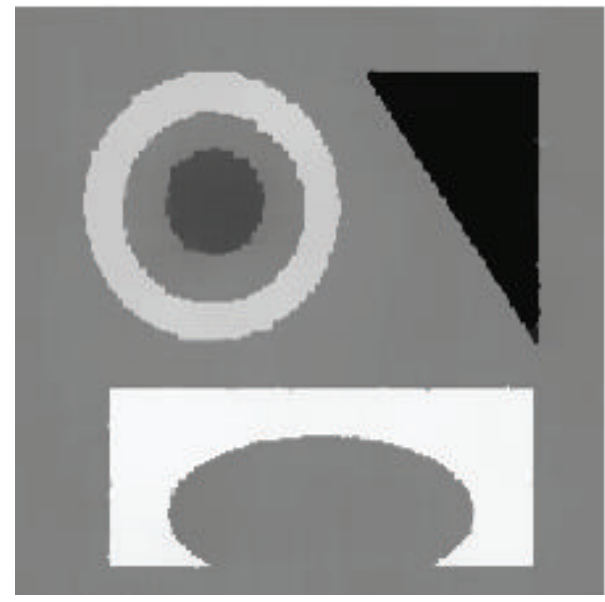

(f)

Figure 1: Synthetic image $(300 \times 300)$. (a) Original image. (b) Noisy image corrupted by Gaussian noise for $\sigma=30$. (c) PM algorithm, $K=4, \tau=0.25$ (356 steps). (d) TV algorithm, $\tau=0.25$ (205 steps). (e) Our method, $K=0.01, \tau=5$ (65 steps). (f) D- $\alpha$-PM algorithm, $k=1, \tau=0.25$, (40 steps). 


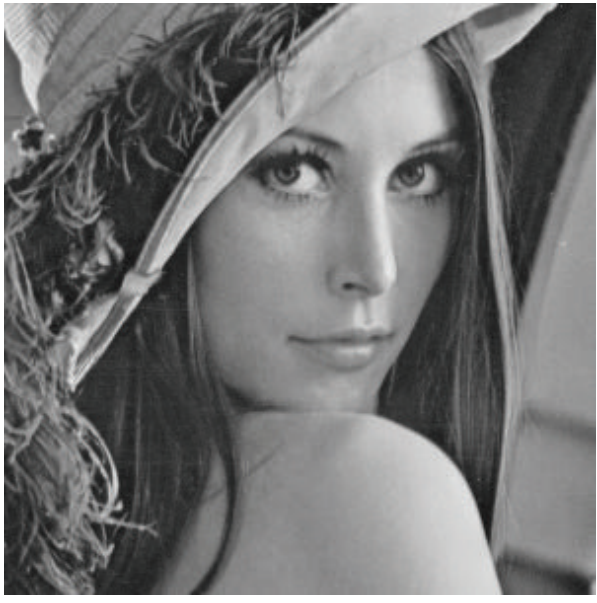

(a)

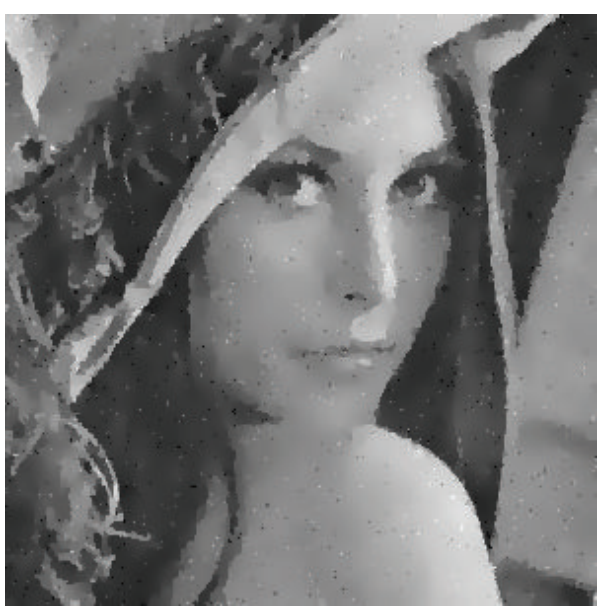

(c)

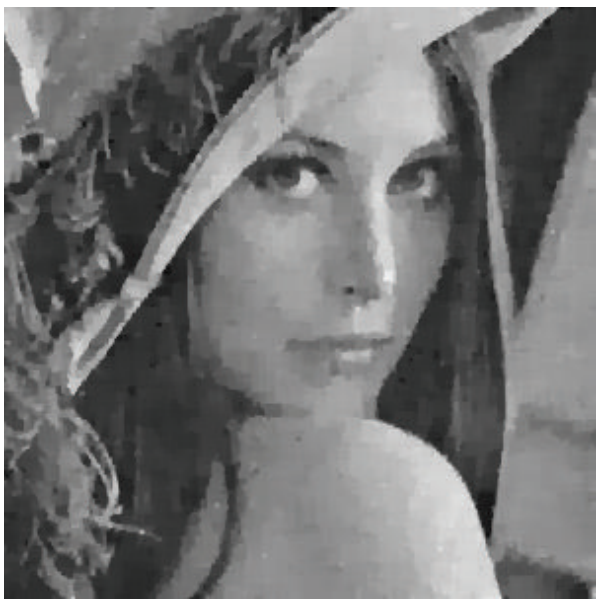

(e)

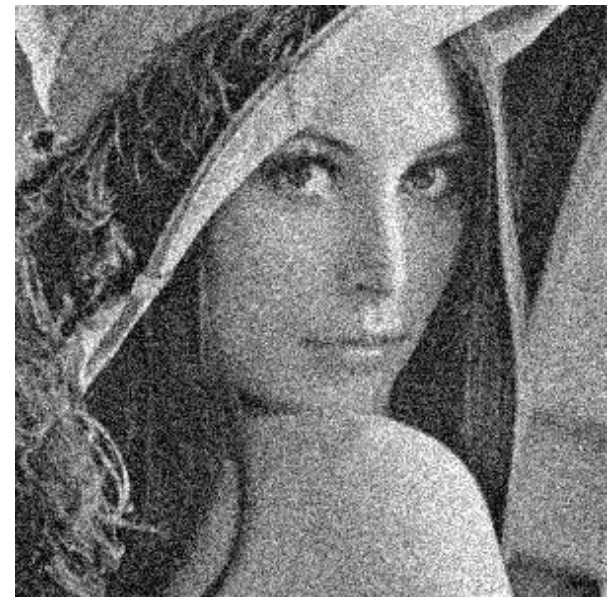

(b)

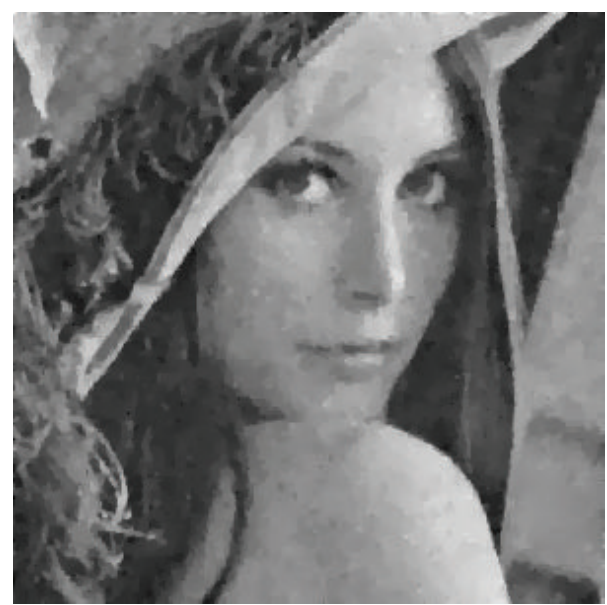

(d)

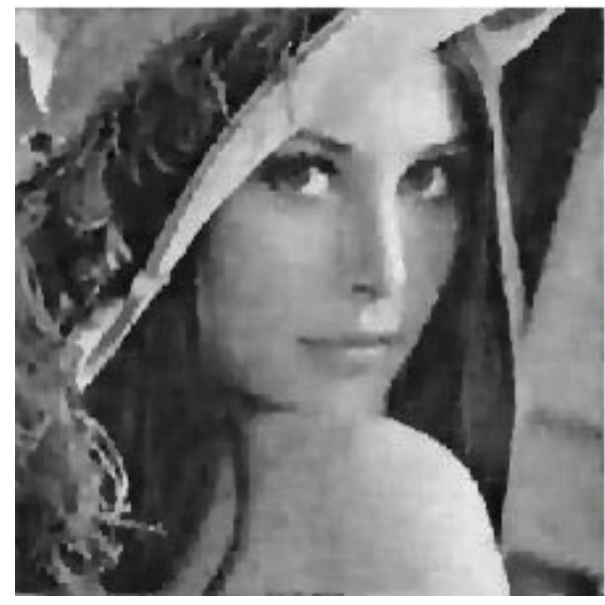

(f)

Figure 2: Lena image $(300 \times 300)$. (a) Original image. (b) Noisy image corrupted by Gaussian noise for $\sigma=30$. (c) PM algorithm, $K=5$, $\tau=0.25$ (113 steps). (d) TV method, $\tau=0.25$ (129 steps). (e) Our method, $K=0.1, \tau=5$ (15 steps). (f) $\mathrm{D}-\alpha$-PM algorithm, $K=4, \tau=2$ (10 steps). 
Consequently, some of the constraints that might affect the practical performance of a regularization formulation modeled along the lines proposed here, including the sample formulation used, include the nature of the noise and even the hardware in which the implementation has been carried out. For more efficient performance, we suggest that platform with higher processing capabilities be deployed. The extent of degradation and the level of preservation required are also other factors that determine the performance of the formulation. For instance, in case of heavy degradation, it is widely recommended that a preconvolution of the noise image with Gaussian be done before the implementation of the suggested formulation to recover edges and other semantically important features. In addition, there may also be cases where noise removal may be counterproductive. This may occur when the oscillation due to noise is of the same scale as that of the features or patterns for which preservation is intended [37]. To surmount such a challenge, one may require a combination of formulations.

However, generally, we have observed that models derived along the lines of the approach proposed here give formulations which not only generate better results but also submit rather progressively to mathematical analysis.

\section{Conflict of Interests}

The authors declare that there is no conflict of interests regarding the publication of this paper.

\section{Acknowledgments}

This work is partially supported by the National Science Foundation of China (11271100 and 11301113), the Ph.D. Programs Foundation of Ministry of Education of China (no. 20132302120057), the class General Financial Grant from the China Postdoctoral Science Foundation (Grant no. 2012M510933), the Fundamental Research Funds for the Central Universities (Grant no. HIT. NSRIF. 2011003), the Program for Innovation Research of Science in Harbin Institute of Technology (Grant no. PIRS OF HIT A201403), and also the 985 Project of Harbin Institute of Technology.

\section{References}

[1] P. Perona and J. Malik, "Scale-space and edge detection using anisotropic diffusion," IEEE Transactions on Pattern Analysis and Machine Intelligence, vol. 12, no. 7, pp. 629-639, 1990.

[2] J. J. Koenderink, “The structure of images," Biological Cybernetics, vol. 50, no. 5, pp. 363-370, 1984.

[3] A. Witkin, Scale-Space Filtering. Readings in Computer Vision: Issues, Problems, Principles, and Paradigms, 1987.

[4] J. Weickert, Anisotropic Diffusion in Image Processing, vol. 1 of European Consortium for Mathematics in Industry, Teubner, Stuttgart, Germany, 1998.

[5] Z. Guo, J. Sun, D. Zhang, and B. Wu, "Adaptive Perona-Malik model based on the variable exponent for image denoising," IEEE Transactions on Image Processing, vol. 21, no. 3, pp. 958967, 2012.
[6] Y. Chen and T. Wunderli, "Adaptive total variation for image restoration in BV space," Journal of Mathematical Analysis and Applications, vol. 272, no. 1, pp. 117-137, 2002.

[7] L. Vese, "A study in the BV space of a denoising-deblurring variational problem," Applied Mathematics and Optimization, vol. 44, no. 2, pp. 131-161, 2001.

[8] L. I. Rudin, S. Osher, and E. Fatemi, "Nonlinear total variation based noise removal algorithms," Physica D: Nonlinear Phenomena, vol. 60, no. 1-4, pp. 259-268, 1992.

[9] S. Osher and L. I. Rudin, "Feature-oriented image enhancement using shock filters," SIAM Journal on Numerical Analysis, vol. 27, no. 4, pp. 919-940, 1990.

[10] G. Aubert and P. Kornprobst, Mathematical Problems in Image Processing: Partial Differential Equations and the Calculus of Variations, vol. 147 of Applied Mathematical Sciences, Springer, New York, NY, USA, 2nd edition, 2006.

[11] A. Marquina and S. Osher, "Explicit algorithms for a new time dependent model based on level set motion for nonlinear deblurring and noise removal," SIAM Journal on Scientific Computing, vol. 22, no. 2, pp. 387-405, 2000.

[12] Y. Chen, S. Levine, and M. Rao, "Variable exponent, linear growth functionals in image restoration," SIAM Journal on Applied Mathematics, vol. 66, no. 4, pp. 1383-1406, 2006.

[13] P. Blomgren, T. F. Chan, and P. Mulet, "Extensions to total variation denoising," in Advanced Signal Processing: Algorithms, Architectures and Implementations VII, Proceedings of the SPIE 3162, pp. 367-375, San Diego, Calif, USA, July 1997.

[14] T. F. Chan and S. Esedoglu, "Aspects of total variation regularized $L^{1}$ function approximation," SIAM Journal on Applied Mathematics, vol. 65, no. 5, pp. 1817-1837, 2005.

[15] D. M. Strong and T. F. Chan, "Spatially and scale adaptive total variation based regularization and anisotropic diffusion in image processing," Diusion in Image Processing, UCLA Math Department CAM Report Cite- seer 1996, 1996.

[16] A. Chambolle and P.-L. Lions, "Image recovery via total variation minimization and related problems," Numerische Mathematik, vol. 76, no. 2, pp. 167-188, 1997.

[17] L. Xiao, L.-L. Huang, and B. Roysam, "Image variational denoising using gradient fidelity on curvelet shrinkage," Eurasip Journal on Advances in Signal Processing, vol. 2010, Article ID 398410, 2010.

[18] T. F. Chan and J. Shen, "Mathematical models for local nontexture inpaintings," SIAM Journal on Applied Mathematics, vol. 62, no. 3, pp. 1019-1043, 2002.

[19] L. Vese, Problemes variationnels et EDP pour l analyse dimages et l evolution de courbes [Ph.D. thesis], Universite de Nice SophiaAntipolis, 1996.

[20] T. Chan, A. Marquina, and P. Mulet, "High-order total variation-based image restoration," SIAM Journal on Scientific Computing, vol. 22, no. 2, pp. 503-516, 2000.

[21] M. Bildhauer and M. Fuchs, "A variational approach to the denoising of images based on different variants of the TV-regularization," Applied Mathematics and Optimization, vol. 66, no. 3, pp. 331-361, 2012.

[22] S. Osher, M. Burger, D. Goldfarb, J. Xu, and W. Yin, "An iterative regularization method for total variation-based image restoration," Multiscale Modeling \& Simulation, vol. 4, no. 2, pp. 460-489, 2005.

[23] X. Zhou, "An evolution problem for plastic antiplanar shear," Applied Mathematics and Optimization, vol. 25, no. 3, pp. 263285, 1992. 
[24] B. Dacorogna, Introduction to the Calculus of Variations, World Scientific, 2004.

[25] M. Xu and S. Zhou, "Existence and uniqueness of weak solutions for a generalized thin film equation," Nonlinear Analysis: Theory, Methods \& Applications, vol. 60, no. 4, pp. 755-774, 2005.

[26] F. Andreu, V. Caselles, J. I. Díaz, and J. M. Mazón, “Some qualitative properties for the total variation flow," Journal of Functional Analysis, vol. 188, no. 2, pp. 516-547, 2002.

[27] Q. Liu, Z. Yao, and Y. Ke, "Entropy solutions for a fourth-order nonlinear degenerate problem for noise removal," Nonlinear Analysis: Theory, Methods \& Applications, vol. 67, no. 6, pp. 1908-1918, 2007.

[28] Z. Wu, J. Zhao, J. Yin, and H. Li, Nonlinear Diffusion Equations, World Scientific, River Edge, NJ, USA, 2001.

[29] Z. Wu, J. Yin, and C. Wang, Elliptic \& Parabolic Equations, World Scientific, River Edge, NJ, USA, 2006.

[30] L. C. Evans and Y. Yu, "Various properties of solutions of the infinity-Laplacian equation," Communications in Partial Differential Equations, vol. 30, no. 7-9, pp. 1401-1428, 2005.

[31] Z. Guo, J. Yin, and Q. Liu, "On a reaction-diffusion system applied to image decomposition and restoration," Mathematical and Computer Modelling, vol. 53, no. 5-6, pp. 1336-1350, 2011.

[32] L. C. Evans and R. F. Gariepy, Measure Theory and Fine Properties of Functions, vol. 5 of Studies in Advanced Mathematics, CRC Press, Boca Raton, Fla, USA, 1992.

[33] J. Weickert, B. M. Ter Haar Romeny, and M. A. Viergever, "Efficient and reliable schemes for nonlinear diffusion filtering," IEEE Transactions on Image Processing, vol. 7, no. 3, pp. 398-410, 1998.

[34] S. Durand, J. Fadili, and M. Nikolova, "Multiplicative noise removal using L1 fidelity on frame coefficients," Journal of Mathematical Imaging and Vision, vol. 36, no. 3, pp. 201-226, 2010.

[35] Z. Wang, A. C. Bovik, H. R. Sheikh, and E. P. Simoncelli, "Image quality assessment: from error visibility to structural similarity," IEEE Transactions on Image Processing, vol. 13, no. 4, pp. 600612, 2004.

[36] A. Horé and D. Ziou, "Image quality metrics: PSNR vs. SSIM," in Proceedings of the 20th International Conference on Pattern Recognition (ICPR '10), pp. 2366-2369, Istanbul, Turkey, August 2010.

[37] Y. Yu and S. T. Acton, "Speckle reducing anisotropic diffusion," IEEE Transactions on Image Processing, vol. 11, no. 11, pp. 12601270, 2002. 


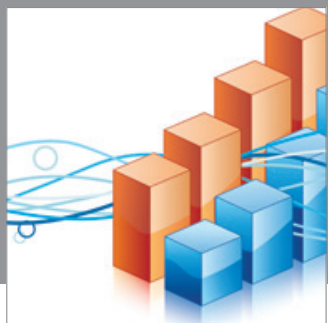

Advances in

Operations Research

mansans

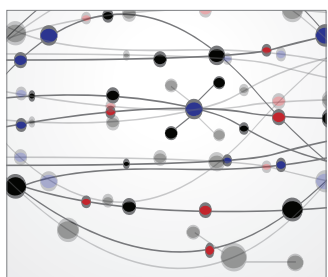

The Scientific World Journal
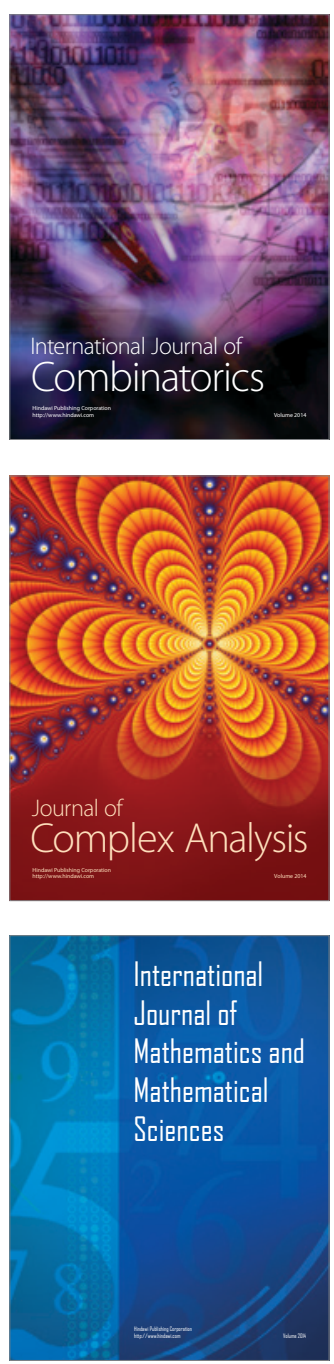
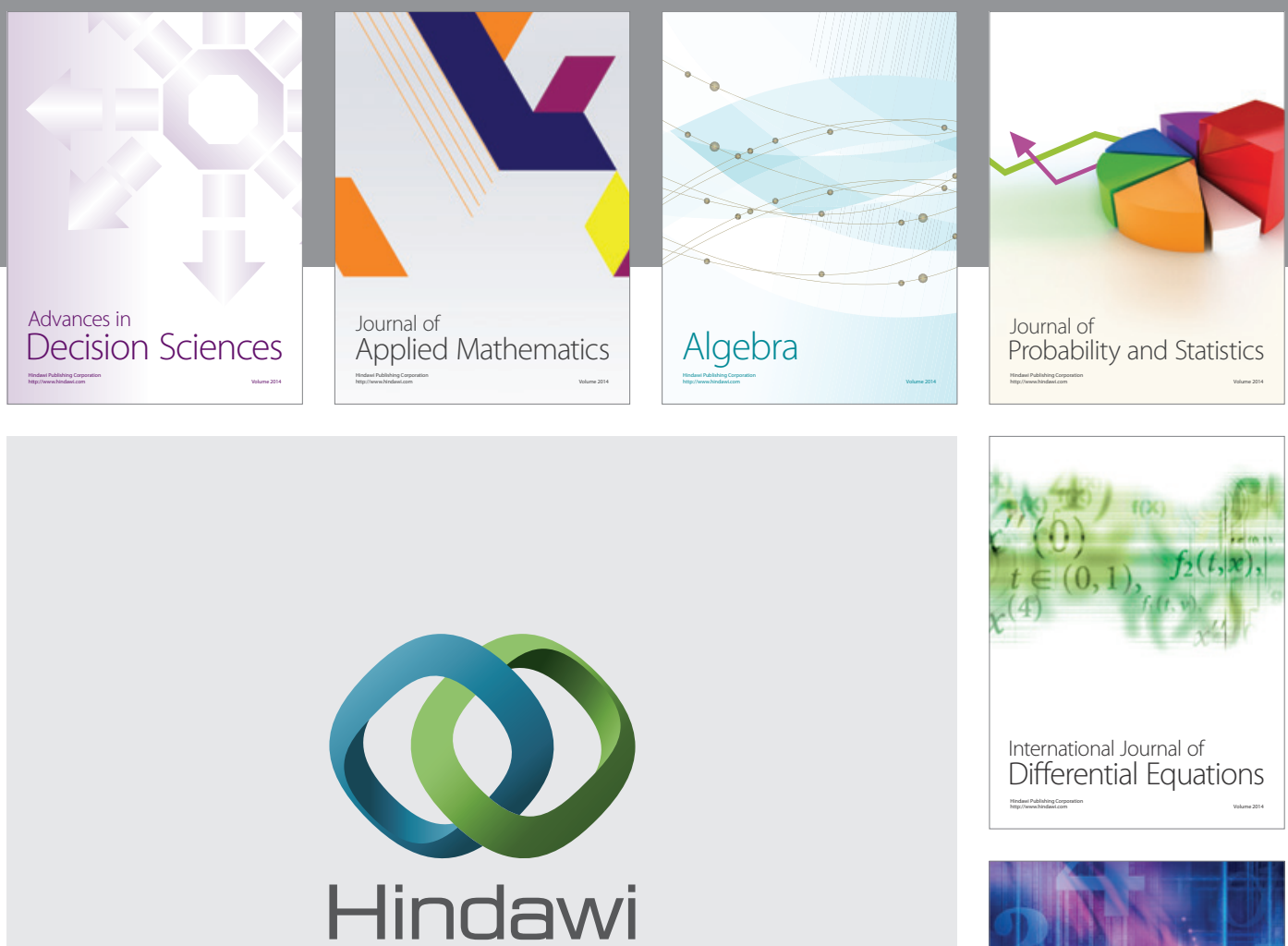

Submit your manuscripts at http://www.hindawi.com
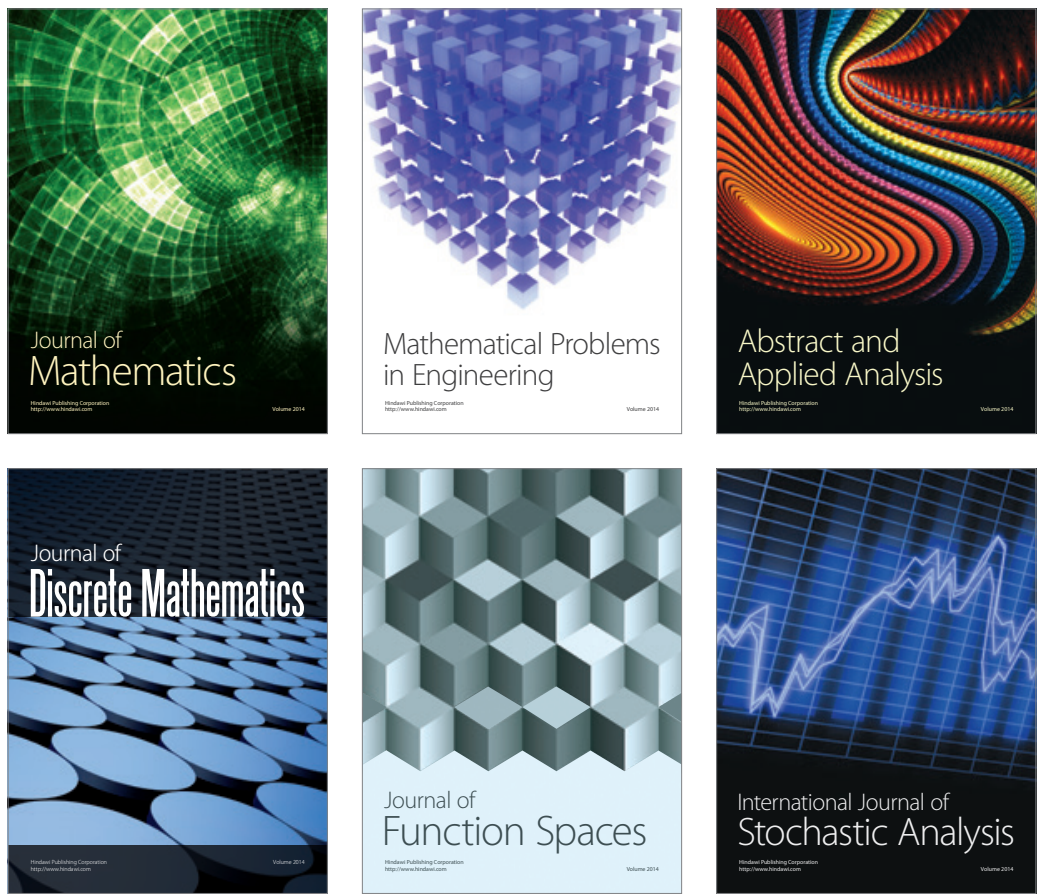

Journal of

Function Spaces

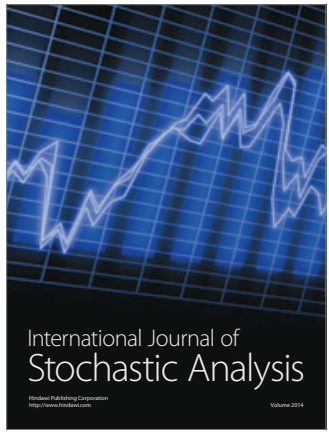

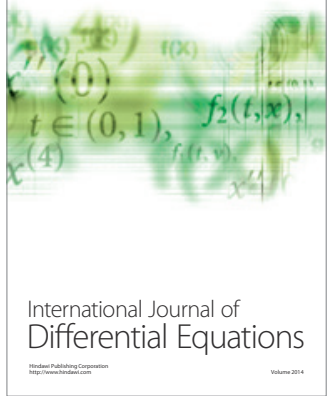
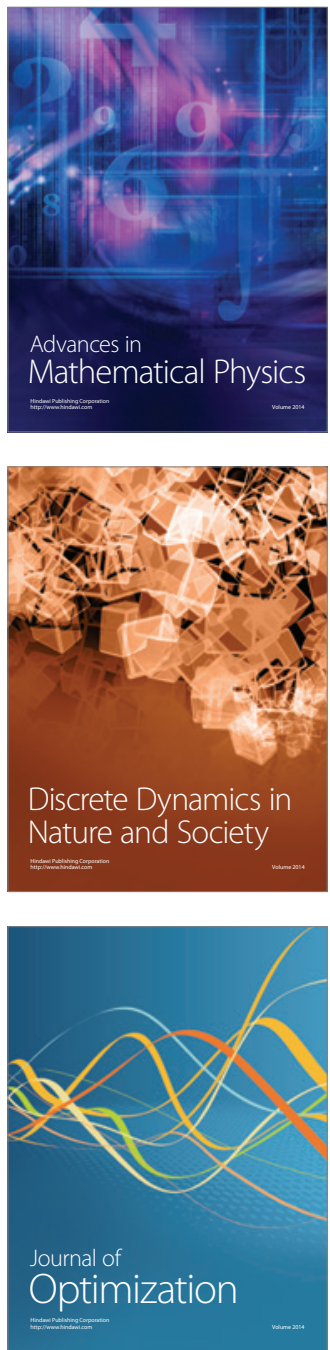